\title{
TENABLE STRATEGY BLOCKS AND SETTLED EQUILIBRIA*
}

\author{
ROGER MYerson ${ }^{\dagger}$ AND JÖRGEN WEIBULL ${ }^{\ddagger}$
}

September 3, 2012. This version: November 28, 2014.

\begin{abstract}
When people interact in familiar settings, social conventions usually develop so that people tend to disregard alternatives outside the convention. For rational players to usually restrict attention to a block of conventional strategies, no player should prefer to deviate from the block when others are likely to act conventionally and rationally inside the block. We explore two set-valued concepts, coarsely and finely tenable blocks, that formalize this notion for finite normal-form games. We then identify settled equilibria, which are Nash equilibria with support in minimal tenable blocks. For a generic class of normal-form games, our coarse and fine concepts are equivalent, and yet they differ from standard solution concepts on open sets of games. We demonstrate the nature and power of the solutions by way of examples. Settled equilibria are closely related to persistent equilibria but are strictly more selective on an open set of games. With fine tenability, we obtain invariance under the insertion of a subgame with a unique totally mixed payoff-equivalent equilibrium, a property that other related concepts have not satisfied.
\end{abstract}

Keywords: Settled equilibrium, tenable block, consideration set, convention, norm.

JEL-codes: C70, C72, C73, D01, D02, D03.

*Earlier versions of this manuscript, with the title "Settled equilibria", have been presented in seminars at the University of Chicago, Ecole Polytechnique, Yale University, and at the KTH Royal Institute of Technology in Stockholm, as well as at the GAMES 2012 world congress, the conference in Jerusalem (June 2013) in honor of Jean-Francois Mertens, at the "Norms, Actions and Games" conference at Imperial College London (April 2014), at the "70 years of Theory of Games and Economic Behavior" conference at the Tinbergen Institute in Amsterdam (June 2014), and at the 25th International Game Theory Conference in Stony Brook (July 2014). We thank Jun Chen, Erik Mohlin, Larry Samuelson, Olivier Tercieux and Troels Sørensen for helpful comments to earlier drafts.

$\dagger$ Department of Economics, University of Chicago.

${ }^{\ddagger}$ Department of Economics, Stockholm School of Economics; Institute for Advanced Studies in Toulouse; Department of Mathematics, KTH Royal Institute of Technology, Stockholm. 


\section{INTRODUCTION}

Schelling (1960) pointed out the importance and subtlety of pure coordination problems, that is, problems in which the participants have common interests but there are multiple ways to coordinate. Sometimes one of the solutions may be "salient" (Schelling, 1960). However, in many situations we must in practice rely on what Lewis (1969) calls precedent in order to solve coordination problems. If all participants know that a particular coordination problem has been solved in a particular way many times before, and this is common knowledge, then this may help them solve a current coordination problem.

More generally, consider a large population that plays familiar games, not necessarily coordination games, in a historical and cultural context where individuals know how similar games have been played in the past. When people interact in such settings, social norms or conventions usually develop, specifying which actions or decision alternatives individuals are expected to, or should, consider. ${ }^{1}$ Such informal institutions, norms or conventions develop over time and people tend to disregard alternatives that are physically available to them but fall outside the norm or convention. Arguably, this is a pervasive phenomenon in all societies. Social institutions are sustained in a larger natural interactive context by viewing such unconventional actions as "illegal" (Hurwicz, 2008). Conformity with social norms helps simplify people's decision-making and coordination. When people are generally expected to act rationally within the conventional norms, unconventional alternatives should not be advantageous. ${ }^{2}$

We here elaborate a theory which permits the endogenous formation of such conventions in finite games given in normal form. A block in such a game is a non-empty set of pure strategies for each player role. We view a block as a potential norm or convention, a candidate for what strategies individuals are likely to seriously consider when called upon to play the game in their player role. The associated block game is the restricted game in which all players are confined to their block strategies. A robustness requirement on a block to be a potential convention is that nobody should be able to do better by choosing a strategy outside the block when others are very likely to use strategies in the block. Any absorbing block (Kalai and Samet, 1984) or any curb block (Basu and Weibull, 1991) meets this robustness requirement. However, such blocks are sometimes very large, and, moreover, may depend on aspects that arguably should be regarded strategically inessential. This is why we here ex-

\footnotetext{
${ }^{1}$ By a convention we mean a pattern of behavior that is customary, expected, and self-enforcing, see Lewis (1969) and Young (1993,1998).

${ }^{2}$ The idea to embed a strategic interaction in a larger societal context is not new to game theory. In his Ph.D. thesis, John Nash mentioned such a perspective, his so-called mass-action interpretation in which there for each player role in the game is a population of boundedly rational individuals (Nash, 1950).
} 
plore weaker block properties, coarse and fine tenability, that are arguably sufficient for maintaining conventions and yet less sensitive to details.

We call blocks that satisfy a slightly weaker robustness requirement coarsely tenable. The weakening is that this robustness should hold at least when the overall population play constitutes an equilibrium. People tend to forget or disregard unused strategies, and minimality of the block allows players to disregard as many unused pure strategies as possible. This simplifies the convention and saves on players' cognitive costs. ${ }^{3}$ A coarsely settled equilibrium is any Nash equilibrium (of the whole game) with support in a minimal coarsely tenable block (that is, one that does not contain any other coarsely tenable block). Minimality can also be viewed as requirement of "internal stability". For while "external stability" could mean that no player should be able to gain by deviating from the block when others are likely to act conventionally and rationally (but may occasionally act unconventionally and/or irrationally), "internal stability" would mean that the block does not properly contain any subblock that, by itself, is externally stable. ${ }^{4}$ So in a sense, minimal coarsely tenable blocks exhibit both a form of "internal" and "external" stability.

Coarse tenability imposes no constraint on "non-conventional" players, those who consider other strategy subsets than the conventional block. In other words, if player types specify what strategy subsets players consider when choosing their strategy, robustness is required under any probability distribution over player types that assigns sufficient probability on the conventional types. However, given the emphasis that game theory traditionally places on rationality, a relevant restriction on such type distributions would be that, among the unconventional types, "more rational" types should be much more prevalent than "less rational" types. Arguably, a "more rational" decision-maker considers more options before making a decision than a "less rational" decision-maker. ${ }^{5}$ We accordingly study with particular interest those type distributions that assign much less probability to one player type than another if the second type only considers a (strict) subset of the strategies considered by the first. We call a block finely tenable if there is no strategy outside the block that would be a better reply for such "rationality biased" type distributions. Since we impose additional assumptions on what individuals are likely to consider when not conventional, every coarsely tenable block is, a fortiori, also finely tenable. Under

\footnotetext{
${ }^{3}$ See e.g. Halpern and Pass (2009) and their references.

${ }^{4}$ See the discussion of stable sets in von Neumann and Morgernstern (1944): “... the rules of rational behavior must provide definitely for the possibility of irrational conduct on the part of others" (4.1.2), "... it appears that the sets of imputations which we are considering correspond to the 'standards of behavior' connected with a social organization" (4.6.1), and "Thus our solutions S correspond to such 'standards of behavior' as have an inner stability: once they are generally accepted they overrule everything else and no part of them can be overruled within the limits of the accepted standards." (4.6.2).

${ }^{5}$ See Remark 3 for a weakinging of this monotonicity requirement.
} 
any "rationality $\varepsilon$-biased" type distribution with respect to a finely tenable block, any Nash equilibrium at the population level induces an $\varepsilon$-proper strategy profile in the given game. We accordingly define a finely settled equilibrium as any proper equilibrium with support in a minimal finely tenable block.

The four mentioned block properties are nested: curb implies absorbing, absorbing implies coarsely tenable, and coarsely tenable implies finely tenable. So fine tenability admits the most blocks, but this implies that it yields the smallest minimal admissible blocks.

As shown in Ritzberger and Weibull (1995), every curb block contains a hyperstable set and hence the support of a proper equilibrium. Moreover, proper equilibria are known to induce a (realization equivalent) sequential equilibrium in every extensive-form game with the given normal form (van Damme, 1984). In other words, the most stringent block property, curb, is consistent with equilibrium theory in a very refined form. We show that even the least stringent block property considered here, that of fine tenability, also has this property.

While the notions of coarse and fine tenability in general differ, they in fact coincide for generic normal-form games. By contrast, while Nash equilibria are generically perfect and proper, this is not true for coarsely and finely settled equilibria. The latter, while being generically identical, constitute a strict subset of the Nash, perfect, proper and persistent equilibria, respectively, in an open set of normal-form games.

Before entering the analysis, let us briefly consider a simple coordination game,

$$
\begin{array}{ccc} 
& L & R \\
L & \alpha, \beta & 0,0 \\
R & 0,0 & \gamma, \delta
\end{array}
$$

where $\alpha, \beta, \gamma, \delta>0$. Such a game has three Nash equilibria; two pure and strict and one mixed. All three are proper equilibria and, when viewed as singleton sets, each of them is also strategically stable in the sense of Kohlberg and Mertens (1986). If the game is played only once by rational players, in the absence of a cultural, historical or social context, the mixed equilibrium may be a reasonable prediction. Indeed, in these games any strategy profile is rationalizable and thus compatible with common knowledge of the game and the players' rationality (Bernheim, 1984; Pearce, 1984; Brandenburger and Dekel, 1987; Tan and Werlang, 1988). Hence, when played once, common knowledge of the game and the players' rationality has no predictive power. However, if such a game is often played in culturally familiar settings, the mixed equilibrium appears very unlikely. One would expect individuals to develop an understanding that coordinates their expectations at one of the strict equilibria. This intuition is captured by the solution concepts developed here and also by persistent equilibrium. However, in other games, our solutions differ from persistence and this is true even under arguably minor elaborations of the game (1), such as when one or 
both of the zero-payoff outcomes is replaced by a zero-sum game; then persistence accepts the mixed equilibrium while the present approach still rejects it. We also show that our approach rejects the mixed equilibrium strategy in game (1) even if this mixed strategy is represented as a third pure strategy. Hence, the rejection does not depend on our minimality requirement per se.

The rest of the paper is organized as follows. Notation and established definitions are given in Section 2. Our model of player types and consideration sets is developed in Section 3. Coarsely tenable blocks and coarsely settled equilibria are introduced in Section 4, while finely tenable blocks and finely settled equilibria are defined and studied in Section 5. In Section 6 we shows that coarsely and finely tenable blocks generically coincide. The nature and power of tenability and settledness is demonstrated in examples throughout the text, but Section 7 provides additional examples that show how the present solutions may differ significantly from established solutions in important classes of games. Section 8 concludes.

\section{PRELIMINARIES}

We consider finite normal-form games $G=\langle N, S, u\rangle$, where $N=\{1, \ldots, n\}$ is the set of players, $S=\times_{i \in N} S_{i}$ is the non-empty and finite set of pure-strategy profiles, $u: S \rightarrow \mathbb{R}^{n}$ is the combined payoff function, where $u_{i}(s) \in \mathbb{R}$ is $i$ 's payoff under pure-strategy profile $s$. Let $m_{i}$ be the number of elements of $S_{i}$ and let $\Delta\left(S_{i}\right)$ denote the set of mixed strategies available to player $i$ :

$$
\Delta\left(S_{i}\right)=\left\{\sigma_{i} \in \mathbb{R}_{+}^{m_{i}}: \sum_{s_{i} \in S_{i}} \sigma_{i}\left(s_{i}\right)=1\right\} .
$$

A strategy $\sigma_{i} \in \Delta\left(S_{i}\right)$ is totally mixed if it assigns positive probability to all pure strategies. Write $\Delta^{o}\left(S_{i}\right)$ for this subset. Likewise, a strategy profile is totally mixed if all strategies are totally mixed. Let $M(S)=\times_{i \in N} \Delta\left(S_{i}\right)$ denote the set of mixed-strategy profiles on $S$ and let $M^{o}(S)=\times_{i \in N} \Delta^{o}\left(S_{i}\right)$. We extend the domain of each payoff function $u_{i}$ in the usual way from $S$ to $M(S)$ by

$$
u_{i}(\sigma)=\sum_{s \in S}\left[\Pi_{j \in N} \sigma_{j}\left(s_{j}\right)\right] \cdot u_{i}(s) .
$$

We use $u_{i}\left(s_{-i}, s_{i}^{\prime}\right)$ to denote the payoff that player $i$ obtains from pure strategy $s_{i}^{\prime} \in S_{i}$ when everyone else plays according to $s \in S$, and likewise for mixed strategies. Likewise, let $u_{i}\left(\sigma_{-i},\left[s_{i}\right]\right)$ be the (expected) payoff that player $i$ obtains from pure strategy $s_{i} \in S_{i}$ when everyone else plays according to $\sigma \in M(S)$. Two pure strategies, $s_{i}^{\prime}, s_{i}^{\prime \prime} \in S_{i}$, are payoff equivalent if $u\left(s_{-i}, s_{i}^{\prime}\right)=u\left(s_{-i}, s_{i}^{\prime \prime}\right)$ for all $s \in S$. A purely reduced normal form game is a game in which no pure strategies are payoff equivalent. ${ }^{6}$ Two pure strategies, $s_{i}^{\prime}, s_{i}^{\prime \prime} \in S_{i}$, are payoff equivalent for player $i$ if

\footnotetext{
${ }^{6}$ This is also called the semi-reduced normal form, see e.g. van Damme (1991).
} 
$u_{i}\left(s_{-i}, s_{i}^{\prime}\right)=u_{i}\left(s_{-i}, s_{i}^{\prime \prime}\right)$ for all $s \in S$. A pure strategy $s_{i} \in S_{i}$ is weakly dominated if there exists a $\sigma_{i}^{\prime} \in \Delta\left(S_{i}\right)$ such that $u_{i}\left(\sigma_{-i}, \sigma_{i}^{\prime}\right) \geq u_{i}\left(\sigma_{-i},\left[s_{i}\right]\right)$ for all $\sigma \in M(S)$ with strict inequality for some $\sigma \in M(S)$. A Nash equilibrium is any strategy profile $\sigma \in M(S)$ such that

$$
u_{i}\left(\sigma_{-i},\left[s_{i}\right]\right)<\max _{r_{i} \in S_{i}} u_{i}\left(\sigma_{-i},\left[r_{i}\right]\right) \quad \Rightarrow \quad \sigma_{i}\left(s_{i}\right)=0 .
$$

A Nash equilibrium is strict if any unilateral deviation incurs a payoff loss.

Definition 1 [Myerson, 1978]. For any $\varepsilon>0$, a strategy profile $\sigma \in M^{o}(S)$ is $\varepsilon$ proper if

$$
u_{i}\left(\sigma_{-i},\left[s_{i}\right]\right)<u_{i}\left(\sigma_{-i},\left[r_{i}\right]\right) \Rightarrow \sigma_{i}\left(s_{i}\right) \leq \varepsilon \cdot \sigma_{i}\left(r_{i}\right) .
$$

A proper equilibrium is any limit of $\varepsilon$-proper strategy profiles as $\varepsilon \rightarrow 0$.

The proper equilibria constitute a non-empty subset of the Nash equilibria. We next turn to the concepts of a persistent retract and a persistent equilibrium. Every finite game has a persistent retract and a persistent equilibrium.

Definition 2 [Kalai and Samet, 1984]. A retract is any set $X=\times_{i \in N} X_{i}$ such that $\varnothing \neq X_{i} \subseteq \Delta\left(S_{i}\right)$ is closed and convex $\forall i \in N$. A retract $X$ is absorbing if it has a neighborhood $U \subseteq M(S)$ such that for all $\sigma^{\prime} \in U$ :

$$
\max _{\sigma_{i} \in X_{i}} u_{i}\left(\sigma_{-i}^{\prime}, \sigma_{i}\right)=\max _{s_{i} \in S_{i}} u_{i}\left(\sigma_{-i}^{\prime},\left[s_{i}\right]\right) \quad \forall i \in N .
$$

A persistent retract is any minimal absorbing retract. A persistent equilibrium is any Nash equilibrium belonging to a persistent set.

We will use the following terminology and notation: a block is any set $T=\times_{i \in N} T_{i}$ such that $\varnothing \neq T_{i} \subseteq S_{i} \forall i \in N$. The associated block game is the game $G_{T}=\langle N, T, u\rangle$ (with $u$ restricted to $T$ ). We embed its mixed strategies in the full strategy space of the game $G: M(T)=\left\{\sigma \in M(S): \sigma_{i}\left(s_{i}\right)=0 \forall s_{i} \notin T_{i}, \forall i \in N\right\}$. If $T$ is a block, then clearly $M(T)$ is a retract. By a slight abuse of language, we will call a block $T$ absorbing if $M(T)$ is absorbing. A strategy profile $\sigma$ has support in a block $T$ if $\sigma_{i}\left(s_{i}\right)=0$ for all players $i \in N$ and strategies $s_{i} \notin T_{i}$. Write $\sigma(T)$ for the probability that a mixed-strategy profile $\sigma \in M(S)$ puts on a block: $\sigma(T)=\sum_{s \in T}\left[\Pi_{i \in N} \sigma_{i}\left(s_{i}\right)\right]$. Thus $\sigma \in M(T)$ iff $\sigma(T)=1$.

Definition 3. A Nash equilibrium of a block game $G_{T}$ is any strategy profile $\sigma \in M(T)$ such that

$$
\sigma_{i}\left(s_{i}\right)>0 \Rightarrow s_{i} \in \arg \max _{t_{i} \in T_{i}} u_{i}\left(\sigma_{-i},\left[t_{i}\right]\right)
$$


Clearly every block game has at least one Nash equilibrium.

Definition 4 [Basu and Weibull, 1991]. A block $T$ is curb ("closed under rational behavior") if

$$
\arg \max _{s_{i} \in S_{i}} u_{i}\left(\sigma_{-i},\left[s_{i}\right]\right) \subseteq T_{i}
$$

for every strategy profile $\sigma \in M(T)$ and every player $i \in N$.

Every finite game has a minimal curb block. As noted in Ritzberger and Weibull (1995), every curb block is absorbing. ${ }^{7}$ The game in the introduction has two minimal curb blocks, the supports of its two strict equilibria. Hence, the mixed equilibrium is not persistent. However, in a slight elaboration of that game, the only absorbing block is the full pure-strategy space, so persistence then looses all its cutting power on the set of Nash equilibria.

Example 1. Consider the extensive-form game

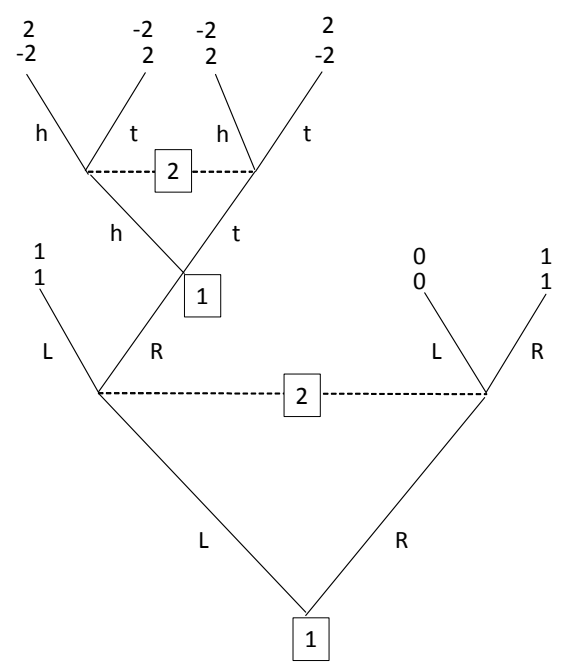

This is an elaboration of game (1) for $\alpha=\beta=\gamma=\delta=1$, where the added subgame is a zero-sum matching-pennies game with value zero. Hence, backward induction requires the players to attach value zero to the subgame, which arguably renders the elaborated game "strategically equivalent" with the original game.

\footnotetext{
${ }^{7}$ To see this, suppose that $T$ is curb. By continuity of payoff functions and the finiteness of the game, there exists a neighborhood $U$ of $M(T) \subseteq M(S)$ such that all mixed-strategy profiles in $U$ have all best replies in the block.
} 
The purely reduced normal-form representation of the elaboration is

$$
\begin{array}{ccccc} 
& & L & R h & R t \\
\text { Game 1: } & L h & 1,1 & 2,-2 & -2,2 \\
& L t & 1,1 & -2,2 & 2,-2 \\
& R & 0,0 & 1,1 & 1,1
\end{array}
$$

This game has three Nash equilibrium components: $A, B$ and $C=\left\{\sigma^{m}\right\}$, where $A$ consists of all strategy profiles of the form $\sigma=(p[L h]+(1-p)[L t],[L])$ for $1 / 4 \leq$ $p \leq 3 / 4, B$ consists of all strategy profiles of the form $\sigma=([R], q[R h]+(1-q)[R t])$ for $1 / 4 \leq q \leq 3 / 4$, and

$$
\left.\sigma^{m}=\left(\frac{1}{2}[R]+\frac{1}{4}[L h]+\frac{1}{4}[L t]\right), \frac{1}{2}[L]+\frac{1}{4}[R h]+\frac{1}{4}[R t]\right) .
$$

There are three proper equilibria: $\sigma^{a}=\left(\frac{1}{2}[L h]+\frac{1}{2}[L t],[L]\right) \in A, \sigma^{b}=\left([R], \frac{1}{2}[R h]+\right.$ $\left.\frac{1}{2}[R t]\right) \in B$, and $\sigma^{m}$. The only absorbing retract is $M(S)$, so $S$ is the only curb set and all Nash equilibria are persistent. The last conclusion is valid for all games (1) with $(\alpha, \beta, \gamma, \delta)$ in an open set containing $(1,1,1,1)$.

The mixed equilibrium $\sigma^{m}$ in this example would arguably be non-robust as a convention, since individuals would presumably learn to avoid the zero-sum subgame and instead be likely to end up in an equilibrium component that corresponds to a strict equilibrium in the original game (1). The solution concepts to be developed below formalize such intuitions.

Remark 1. Other related ideas in the recent literature are so-called prep sets (Voorneveld 2004, 2005) and p-best response sets (Tercieux, 2006 a,b). A prep set (or preparation) is a block $T$ that contains at least one best reply for each player to every mixed strategy on the block. Every pure Nash equilibrium (viewed as a singleton block) is thus a prep set and every curb set is a prep set. Voorneveld (2004) shows that minimal prep sets generically coincide with minimal curb sets and Voorneveld (2005) establishes that prep sets also generically coincide with persistent retracts ( $T$ being a prep set and $M(T)$ an absorbing retract). Tercieux (2006a) defines a p-best response set as a block that contains all best replies to all beliefs that put at least probability $p$ on the block, where beliefs are not constrained to treat other players' strategy choices as statistically independent (a constraint we here impose). Tercieux (2006b) weakens the requirement "all best replies" to "some best reply," and calls the first notion strict $p$-best response sets. For all finite two-player games: (a) any strict p-best response set with $p<1$ is curb, and every curb set is a strict p-best response set for some $p<1$ (see Lemma 2 in Ritzberger and Weibull, 1995), and (b) if a block $T$ is a (weak) $p$-best response set with $p<1$, then $M(T)$ is an absorbing retract, 
and if $M(T)$ is an absorbing retract, then $T$ is a (weak) $p$-best response set for some $p<1$. It follows that $T$ is a minimal (weak) p-best response set for some $p<1$ if and only if $X=M(T)$ is a persistent set.

A second related idea appears in some papers in epistemic game theory. See Brandenburger, Friedenberg and Keisler (2008), Brandenburger and Friedenberg (2010), and Battigalli and Friedenberg (2012). In these studies, players face uncertainty about the play of the game but interactions take place in social contexts where conventions may form, and these conventions can influence participants' beliefs about what others will do (and not do). However, in that framework beliefs may be incorrect, while we here assume that participants' beliefs are correct at the population level. Hence, the solution concepts are distinct and predictions differ.

A third related idea is the refined best-response correspondence in Balkenborg, Hofbauer, and Kuzmics (2013,2014). Their correspondence shares many properties with the usual best-response correspondence- such as being upper hemi-continuous, closed- and convex-valued - but generically differs in games with more than two players. However, in two-player normal form games it generically coincides with the usual best-response correspondence. Hence, their solutions generically differ from ours.

\section{Consideration-Set GAMes}

We proceed to construct a framework within which one can make precise the idea that conventions or norms should be such that when people are generally expected to act rationally within the convention or norm at hand, unconventional alternatives should not be advantageous. We do this in terms of a situation in which individuals are very likely to consider only the strategies in some conventional block, but allowing for the possibility that some individuals may also consider strategies outside the block. An individual's effective strategy set, to be called his or her consideration set, a nonempty subset of the full strategy set, will be treated as his or her type in a game of incomplete information where types are private information. ${ }^{8}$

More precisely, let $G=\langle N, S, u\rangle$ be a finite game. As in Nash's mass action interpretation, let there for each player role $i \in N$ be a large population of individuals who are now and then randomly called upon to play the game $G$ in that player role. Let the type space for each player role $i \in N$ be $\Theta_{i}=\mathcal{C}\left(S_{i}\right)$, where $\mathcal{C}\left(S_{i}\right)$ denotes the collection of non-empty subsets $C_{i}$ of $S_{i}$. Let $\mu_{i}$ be any probability distribution over $\mathcal{C}\left(S_{i}\right)$, where $\mu_{i}\left(C_{i}\right) \in[0,1]$ is the probability that the individual drawn to play in role $i$ will be of type $\theta_{i}=C_{i}$, that is, have $C_{i}$ as his or her consideration set. These random draws of types, one draw for each player population, are statistically independent.

\footnotetext{
${ }^{8}$ The term "consideration set" is borrowed from management science and marketing. The basic idea is that decision-makers may not consider all choices available to them. The term originates with Wright and Barbour (1977). For recent contributions to this literature, see Manzini and Mariotti (2007, 2014), Salant and Rubinstein (2008), and Eliaz and Spiegler (2011).
} 
A vector $\mu=\left(\mu_{1}, . ., \mu_{n}\right) \in \times_{i \in N} \Delta\left(\mathcal{C}\left(S_{i}\right)\right)$ is thus a type distribution, where the probability that any given block $T=\times_{i \in N} T_{i}$ will be the actual consideration block is the product of the probabilities for each player role; $\mu_{1}\left(T_{1}\right) \cdot \ldots \mu_{n}\left(T_{n}\right)$. With some abuse of notation, we will write $\mu(T)$ for this product probability.

Each type distribution $\mu$ defines a game $G^{\mu}=\left\langle N, F, u^{\mu}\right\rangle$ of incomplete information in which a pure strategy for each player role $i \in N$ is a function $f_{i}: \mathcal{C}\left(S_{i}\right) \rightarrow S_{i}$ such that $f_{i}\left(C_{i}\right) \in C_{i}$ for all $C_{i} \in \mathcal{C}\left(S_{i}\right)$. In other words, a pure strategy $f_{i}$ prescribes for each type $\theta_{i} \in \Theta_{i}$ a pure strategy in the type's consideration set $C_{i}$. Let $F_{i}$ be the set of such functions and write $F=\times_{i \in N} F_{i}$. Each pure-strategy profile $f \in F$ induces a mixed-strategy profile $\sigma^{f, \mu} \in M(S)$ in $G$, where the probability that player $i \in N$ will use pure strategy $s_{i} \in S_{i}$ is

$$
\sigma_{i}^{f, \mu}\left(s_{i}\right)=\sum_{C_{i} \in \mathcal{C}\left(S_{i}\right)} \mu_{i}\left(C_{i}\right) \cdot \mathbf{1}_{f_{i}\left(C_{i}\right)=s_{i}} .
$$

The resulting expected payoff to each player $i$ is $u_{i}^{\mu}(f)=u_{i}\left(\sigma^{f, \mu}\right)$. This defines the payoff functions $u_{i}^{\mu}: F \rightarrow \mathbb{R}$ for all players $i \in N$ in $G^{\mu}$. The consideration-set game $G^{\mu}$, so defined, is finite. Payoffs to mixed-strategy profiles can be defined in the usual way. By Nash's existence theorem, each consideration-set game $G^{\mu}$ has at least one Nash equilibrium in pure or mixed strategies. ${ }^{9}$

For any mixed-strategy profile $\tau \in M(F)$, player role $i \in N$ and strategy subset $C_{i} \in \mathcal{C}\left(S_{i}\right)$, let $\tau_{i \mid C_{i}} \in \Delta\left(S_{i}\right)$ be the conditional probability distribution over the strategy set $S_{i}$, given that $\theta_{i}=C_{i}$ is $i$ 's type (in particular, $\tau_{i \mid C_{i}}\left(s_{i}\right)=0 \forall s_{i} \notin C_{i}$ ). When a mixed-strategy profile $\tau \in M(F)$ is played in $G^{\mu}$, pure strategy $s_{i} \in S_{i}$ will be used with probability

$$
\tau_{i}^{\mu}\left(s_{i}\right)=\sum_{C_{i} \in \mathcal{C}\left(S_{i}\right)} \mu_{i}\left(C_{i}\right) \cdot \tau_{i \mid C_{i}}\left(s_{i}\right) .
$$

This defines the mixed-strategy profile $\tau^{\mu} \in M(S)$ induced by $\tau$ in the underlying game $G$. We will sometimes refer to $\tau^{\mu}$ as the projection of $\tau \in M(F)$ to $M(S)$ under the type distribution $\mu$.

A strategy profile $\tau \in M(F)$ is a Nash equilibrium of $G^{\mu}$ if and only if for all player roles $i \in N$ and consideration sets $C_{i} \in \mathcal{C}\left(S_{i}\right)$,

$$
\mu_{i}\left(C_{i}\right)>0 \Rightarrow u_{i}\left(\tau_{-i}^{\mu}, \tau_{i \mid C_{i}}\right)=\max _{s_{i} \in C_{i}} u_{i}\left(\tau_{-i}^{\mu},\left[s_{i}\right]\right) .
$$

It is easily verified that the projections of Nash equilibria of $G^{\mu}$ to $G$ converge to Nash equilibria of $G_{T}$ as $\mu(T) \rightarrow 1$.

\footnotetext{
${ }^{9}$ A special case of this set-up is when $\mu(S)=1$. Then $G^{\mu}$ is effectively the same as $G$; the probability is then one that all players will consider all pure strategies at their disposal in $G$.
} 


\section{Coarsely tenable Blocks and COARsely Settled Equilibria}

Let $G=\langle N, S, u\rangle$ be any finite game and let $T$ be any block, interpreted as a potential convention. Call an individual in player population $i \in N$ conventional if his or her type is $\theta_{i}=T_{i}$. The following definition formalizes the robustness requirement on such a convention that if the type distribution $\mu$ is such that individuals are very likely to be conventional, and if their average play would constitute a Nash equilibrium of the associated game of incomplete information, $G^{\mu}$, then nobody could do better by choosing a strategy outside the block.

Definition 5. A block $T$ is coarsely tenable if there exists an $\varepsilon \in(0,1)$ such that

$$
\max _{t_{i} \in T_{i}} u_{i}\left(\tau_{-i}^{\mu},\left[t_{i}\right]\right)=\max _{s_{i} \in S_{i}} u_{i}\left(\tau_{-i}^{\mu},\left[s_{i}\right]\right) \quad \forall i \in N
$$

for every type distribution $\mu$ with $\mu_{i}\left(T_{i}\right)>1-\varepsilon \forall i \in N$ and every Nash equilibrium $\tau$ of $G^{\mu}$.

Clearly the full block $T=S$ is coarsely tenable in this sense. Also a singleton block that is the support of any pure strict equilibrium is coarsely tenable, and so is any curb block and any absorbing block. To see why the last claim holds, let $T$ be an absorbing block. By definition, there then exists an $\varepsilon \in(0,1)$ such that

$$
\max _{t_{i} \in T_{i}} u_{i}\left(\sigma_{-i},\left[t_{i}\right]\right)=\max _{s_{i} \in S_{i}} u_{i}\left(\sigma_{-i},\left[s_{i}\right]\right) \quad \forall i \in N
$$

if $\sigma \in M(S)$ is such that $\sigma_{i}\left(T_{i}\right)>1-\varepsilon$ for all players $i \in N$. Let $\tau \in M(F)$ be a Nash equilibrium of any consideration-set game $G^{\mu}$ such that $\mu_{i}\left(T_{i}\right)>1-\varepsilon$ for this $\varepsilon$ and for all players $i$. Then $\tau_{i}^{\mu}\left(T_{i}\right)>1-\varepsilon$ for all players $i \in N .{ }^{10}$ Thus (4) holds.

We also note that the equilibria of the block game associated with a coarsely tenable block coincide with the equilibria of the original game that have support in the block. In other words, oblivion of strategies outside a coarsely tenable block comes at no cost. In sum:

Proposition 1. Every absorbing block is coarsely tenable. If a block $T$ is coarsely tenable, then the Nash equilibria of the block game $G_{T}$ are precisely the Nash equilibria of $G$ that have support in $T$.

$$
\begin{aligned}
& { }^{10} \text { By definition, } \\
& \tau_{i}^{\mu}\left(T_{i}\right)=\sum_{t_{i} \in T_{i}} \tau_{i}^{\mu}\left(t_{i}\right)=\sum_{t_{i} \in T_{i}} \sum_{C_{i} \in \mathcal{C}\left(S_{i}\right)} \mu_{i}\left(C_{i}\right) \cdot \tau_{i \mid C_{i}}\left(t_{i}\right) \geq \mu_{i}\left(T_{i}\right) \cdot \sum_{t_{i} \in T_{i}} \tau_{i \mid T_{i}}\left(t_{i}\right)=\mu_{i}\left(T_{i}\right)>1-\varepsilon .
\end{aligned}
$$


Proof: To prove the last claim, let $T$ be any block in any finite game $G$. First, if $\sigma \in M(T)$ is a Nash equilibrium of $G$, it is, a fortiori, a Nash equilibrium of $G_{T}$. Secondly, suppose that $\sigma \in M(T)$ is a Nash equilibrium of $G_{T}$. Let $\mu(T)=1$ and let $\tau \in M(F)$ be such that $\tau_{i \mid T_{i}}=\sigma_{i}$. Then $\tau^{\mu}=\sigma$, and by (3), $\tau$ is a Nash equilibrium of $G^{\mu}$. If $T$ is coarsely tenable:

$u_{i}(\sigma)=u_{i}\left(\tau_{-i}^{\mu}, \tau_{i \mid T_{i}}\right)=\max _{t_{i} \in T_{i}} u_{i}\left(\tau_{-i}^{\mu},\left[t_{i}\right]\right)=\max _{s_{i} \in S_{i}} u_{i}\left(\tau_{-i}^{\mu},\left[s_{i}\right]\right)=\max _{s_{i} \in S_{i}} u_{i}\left(\sigma_{-i},\left[s_{i}\right]\right) \quad \forall i \in N$

\section{Q.E.D.}

Kalai and Samet (1984) show that elimination of weakly dominated strategies and/or payoff-equivalent strategies from the full strategy space $S$ results in an absorbing retract. ${ }^{11}$ Since absorbing blocks are coarsely tenable, qualitatively similar conclusions hold for coarsely tenable blocks. More precisely, for each player $i$ in $G$, let $T_{i} \subseteq S_{i}$ be such that every pure strategy not in $T_{i}$ is weakly dominated by some mixed strategy with support in $T_{i}$. Then $T$ is coarsely tenable, since each player $i$ will have some (globally) best reply in $T_{i}$ to the projection $\tau^{\mu}$ of any mixed-strategy profile $\tau \in M(F)$ in any consideration-set game $G^{\mu}$. Likewise, for each player $i$, let $T_{i} \subseteq S_{i}$ be such that for every pure strategy not in $T_{i}$ there exists a strategy in $T_{i}$ that is payoff-equivalent for player $i$. Then $T$ is coarsely tenable.

Conventions tend to simplify the interaction at hand by focusing on few strategies. Hence, if a block of strategies would start to become a convention and if it would properly contains a subblock that, by itself, would have the same "external stability" property as the initial block, then play would presumably, over time, tend towards one such subblock. This suggests that minimal coarsely tenable blocks are particularly relevant for prediction, that is, coarsely tenable blocks that do not contain other coarsely tenable blocks. The games we study are finite and hence admit at least one such block. The following definition formalizes an equilibrium notion that combines the (simplicity and internal stability) requirement of minimality of the set of conventional strategies with the (rationality and external stability) requirement that individuals should not be able to benefit by using unconventional strategies when others are likely to use conventional strategies and population play is in equilibrium.

Definition 6. A coarsely settled equilibrium is any Nash equilibrium of $G$ that has support in some minimal coarsely tenable block $T$.

Evidently, any pure strict equilibrium is coarsely settled. By contrast, the mixed equilibrium in game (1) is not, since it does not have support in a minimal coarsely

\footnotetext{
${ }^{11}$ Here "payoff equivalent" can be interpreted in the weak sense of payoff equivalence for the player in question (see Section 2).
} 
tenable block. ${ }^{12}$ In that example, also the notion of persistent equilibrium rejects the mixed equilibrium. In the elaborated version of this game, Game 1 in Example 1, the totally mixed Nash equilibrium was seen to be persistent, although one may argue that the elaboration is strategically irrelevant and hence should not affect the set of solutions. However, the totally mixed equilibrium in the elaboration is not coarsely settled. This follows from the fact that Game 1 has two minimal coarsely tenable blocks, associated with each of the two continuum Nash equilibrium components, $A$ and $B$. These blocks are $T^{A}=\{L h, L t\} \times S_{2}$ and $T^{B}=S_{1} \times\{R h, R t\}$. To see that $T^{A}$ is coarsely tenable, note that in all Nash equilibria of the associated block game, $G_{T^{A}}$, player 2 only uses strategy $L$. Hence, since the projections of Nash equilibria of $G^{\mu}$ converge to Nash equilibria of $G_{T^{A}}$ as $\mu\left(T^{A}\right) \rightarrow 1$, strategy $R$ is not a best reply for player 1 in any Nash equilibrium of $G^{\mu}$ when $\mu\left(T^{A}\right)$ is close to 1 , and hence (4) holds for all $\varepsilon>0$ sufficiently small. The coarsely settled equilibria of Game 1 are thus the Nash equilibria in the equilibrium components $A$ and $B$, with outcomes identical with those in the two strict equilibria of the original game (1).

\section{Finely tenable Blocks and finely settled equilibria}

Imposing restrictions on the type distributions in the consideration-set games beyond the requirement that they should attach high probabilities to the conventional types, could allow for smaller blocks - a finer block structure. The following definition formalizes the notion that (a) individuals are very likely to be of the conventional types for the block (as under coarse tenability), (b) all types have positive probability, and (c) unconventional types (those with other consideration sets than those constituting the block) are much more likely to have larger than smaller consideration sets (in terms of set inclusion). In other words, these type distributions place much more probability weight on "more rational types" (who consider more strategic alternatives) than "less rational types" (who consider fewer strategic alternatives). In particular, the most likely among the unconventional types is the "standard" type of player in game theory, the type who considers all strategies available in his or her player role. Formally and more precisely, we now focus on type distributions of the following variety:

Definition 7. For any block $T$ and any $\varepsilon \in(0,1)$, a type distribution $\mu$ is $\varepsilon$-proper on $T$ if

$$
\begin{cases}\text { (a) } & \mu_{i}\left(T_{i}\right)>1-\varepsilon \\ \text { (b) } & \mu_{i}\left(C_{i}\right)>0 \quad \forall C_{i} \in \mathcal{C}\left(S_{i}\right) \\ \text { (c) } & T_{i} \neq C_{i} \subset D_{i} \Rightarrow \mu_{i}\left(C_{i}\right) \leq \varepsilon \cdot \mu_{i}\left(D_{i}\right)\end{cases}
$$

for every player $i \in N$.

\footnotetext{
${ }^{12}$ In Section 7 we show that this conclusion holds also when the mixed equilibrium is represented as a pair of pure strategies added to the game.
} 
The following remark shows that a type distribution has this "rationality bias" property if every unconventional individual's inattention is statistically independent across his or her strategy set.

Remark 2. Let $G=\langle N, S, u\rangle$ be a finite game and let $T$ be a block, interpreted as a potential convention. For all players $i \in N$ and all consideration sets $C_{i}$ other than $T_{i}$, let

$$
\mu_{i}\left(C_{i}\right)=\varepsilon \cdot \Pi_{s_{i} \in C_{i}}\left(1-\delta_{i}\left(s_{i}\right)\right) \cdot \Pi_{s_{i} \notin C_{i}} \delta_{i}\left(s_{i}\right)
$$

(with the last product defined as unity in case $C_{i}=S_{i}$ ). This can be interpreted as follows. For each player role $i \in N$ in the game there is a large population of individuals who are now and then called upon to play the game, just as in Nash's massaction interpretation. The fraction $1-\varepsilon$ of each player population are conventional; their consideration sets are those that define the block. Among the unconventional individuals, who make up the population fraction $\varepsilon \in(0,1)$, each pure strategy $s_{i} \in S_{i}$ is ignored with some probability $\delta_{i}\left(s_{i}\right) \in(0,1)$, and these are statistically independent events for all pure strategies and individuals, hence the formula (5). ${ }^{13}$ Such a type distribution $\mu$ is $\varepsilon$-proper on $T$ if all probabilities $\delta_{i}\left(s_{i}\right)$ are sufficiently small. To see this, let $\left\|\delta_{i}\right\|=\max _{s_{i} \in S_{i}} \delta_{i}\left(s_{i}\right)$. Clearly $\mu_{i}\left(T_{i}\right)>1-\varepsilon$ and $\mu_{i}\left(C_{i}\right)>0$ for all $C_{i} \in \mathcal{C}\left(S_{i}\right)$. Suppose that $C_{i}, D_{i} \in \mathcal{C}\left(S_{i}\right), C_{i} \subset D_{i}$ and $C_{i} \neq T_{i}$. Then

$$
\mu_{i}\left(C_{i}\right) \leq \mu_{i}\left(D_{i}\right) \cdot \prod_{s_{i} \in D_{i} \backslash C_{i}} \frac{\delta_{i}\left(s_{i}\right)}{1-\delta_{i}\left(s_{i}\right)} \leq \frac{\left\|\delta_{i}\right\|}{1-\left\|\delta_{i}\right\|} \cdot \mu_{i}\left(D_{i}\right) .
$$

The factor in front of $\mu_{i}\left(D_{i}\right)$ is less than $\varepsilon$ if $\left\|\delta_{i}\right\|<\varepsilon /(1+\varepsilon) .{ }^{14}$

By requiring robustness only to type distributions that are $\varepsilon$-proper on the block in question, one obtains the following weaker block property:

Definition 8. A block $T$ is finely tenable in $G=\langle N, S, u\rangle$ if there exists an $\varepsilon \in$ $(0,1)$ such that

$$
\max _{t_{i} \in T_{i}} u_{i}\left(\tau_{-i}^{\mu},\left[t_{i}\right]\right)=\max _{s_{i} \in S_{i}} u_{i}\left(\tau_{-i}^{\mu},\left[s_{i}\right]\right) \quad \forall i \in N
$$

holds for every type distribution $\mu$ that is $\varepsilon$-proper on $T$ and every Nash equilibrium $\tau$ of $G^{\mu}$.

\footnotetext{
${ }^{13}$ If an individual would in this way ignore all pure strategies in his player role, then he would "wake up" and consider the conventional set. This follows from (5):

$$
\mu_{i}\left(T_{i}\right)=(1-\varepsilon)+\varepsilon \cdot\left[\Pi_{s_{i} \in T_{i}}\left(1-\delta_{i}\left(s_{i}\right)\right) \cdot \Pi_{s_{i} \notin T_{i}} \delta_{i}\left(s_{i}\right)+\Pi_{s_{i} \in S_{i}} \delta_{i}\left(s_{i}\right)\right]
$$

${ }^{14}$ See Manzini and Mariotti (2013) for decision-theoretic foundations for such statistically independent inattention, and for relations with random-utility models.
} 
Since every coarsely tenable block is, a fortiori, also finely tenable, there are, in general, more finely than coarsely tenable blocks. In particular, minimal finely tenable blocks may be smaller than the minimal coarsely tenable blocks.

Example 1 continued. In each of the two minimal coarsely tenable blocks in Game 1 in Example 1, there are pure strategies not used in any block equilibrium. Player 2 uses only pure strategy $L$ in all block equilibria in $T^{A}$ and player 1 uses only pure strategy $R$ in all block equilibria in $T^{B}$. Nevertheless, for these blocks to be coarsely settled, all 2's strategies need to be included in $T^{A}$ and all 1's strategies in $T^{B}$, since otherwise there will be block equilibria with a better reply outside the block. In particular, the subblock $T^{*}=\{L h, L t\} \times\{L\}$ of the coarsely tenable block $T^{A}$ is not coarsely tenable. However, it is finely tenable. To see this, let $\varepsilon \in(0,1)$ and let $\mu$ be any $\varepsilon$-proper type distribution on $T^{*}$. Consider any Nash equilibrium $\tau \in M(F)$ in the associated consideration-set game $G^{\mu}$. Then $\tau_{1}^{\mu}(L h)=\tau_{1}^{\mu}(L t)$. For suppose that $\tau_{1}^{\mu}(L h)>\tau_{1}^{\mu}(L t)$. By (2) we then have, for $\varepsilon$ sufficiently small, $\tau_{2}^{\mu}(R h)=\mu_{2}(\{R t\})$ and $\tau_{2}^{\mu}(R t) \geq \mu_{2}(\{R h, R t\})$. Since $\mu$ is $\varepsilon$-proper on $T^{*}$, we also have

$$
\mu_{2}(\{R h\}) \leq \varepsilon \cdot \mu_{2}(\{R h, R t\}),
$$

so $\tau_{2}^{\mu}(R h)<\varepsilon \cdot \tau_{2}^{\mu}(R t)$. Then Lt is a best reply for player 1 , and $\tau_{1}^{\mu}(L h)<\tau_{1}^{\mu}(L t)$, a contradiction. By the same token, $\tau_{1}^{\mu}(L h)>\tau_{1}^{\mu}(L t)$ is not possible. A similar argument establishes $\tau_{2}^{\mu}(R h)=\tau_{2}^{\mu}(R t)$. These two equations imply that each player $i$ has a best reply to $\tau^{\mu}$ in $T_{i}^{*}$, that is, (4) holds and $T^{*}$ is finely tenable.

The naming of the considered type distributions is due to the following observation: when a block is finely tenable, the projection of any Nash equilibrium in any consideration-set game $G^{\mu}$ where the type distribution $\mu$ is $\varepsilon$-proper on $T$, constitutes an $\varepsilon$-proper strategy profile in the original game $G$.

Proposition 2. Let $T$ be a finely tenable block and let $\varepsilon$ be as in Definition 8. If $\mu$ is any type distribution that is $\varepsilon$-proper on $T$, and if $\tau \in M(F)$ is a Nash equilibrium of $G^{\mu}$, then $\tau^{\mu} \in M(S)$ is an $\varepsilon$-proper strategy profile in $G$.

Proof: To show that $\tau^{\mu}$ is an $\varepsilon$-proper strategy profile in $G$ we first note that since each $C_{i} \in \mathcal{C}\left(S_{i}\right)$ has positive probability of being the consideration set under $\mu$, $\tau_{i}\left(f_{i}\right)=0$ for all pure strategies $f_{i} \in F_{i}$ such that $f_{i}\left(C_{i}\right) \notin \arg \max _{s_{i} \in C_{i}} u_{i}\left(\tau_{-i}^{\mu},\left[s_{i}\right]\right)$ for some $C_{i} \in \mathcal{C}\left(S_{i}\right)$. Secondly, let $r_{i}, s_{i} \in S_{i}$ be such that $u_{i}\left(\tau_{-i}^{\mu},\left[r_{i}\right]\right)<u_{i}\left(\tau_{-i}^{\mu},\left[s_{i}\right]\right)$ and let $\mathcal{R}_{i} \subseteq \mathcal{C}\left(S_{i}\right)$ be the collection of sets $C_{i} \in \mathcal{C}\left(S_{i}\right)$ such that

$$
r_{i} \in \arg \max _{c_{i} \in C_{i}} u_{i}\left(\tau_{-i}^{\mu},\left[c_{i}\right]\right) .
$$


Clearly $C_{i} \in \mathcal{R}_{i} \Rightarrow s_{i} \notin C_{i}$. Moreover, $T_{i} \notin \mathcal{R}_{i}$, since $T$ is finely tenable and thus contains a pure best reply to $\tau^{\mu}$. For each $C_{i} \in \mathcal{R}_{i}$ :

$$
\left\{s_{i}\right\}=\arg \max _{s_{i}^{\prime} \in C_{i} \cup\left\{s_{i}\right\}} u_{i}\left(\tau_{-i}^{\mu},\left[s_{i}^{\prime}\right]\right),
$$

(iii) $\quad \sum_{C_{i} \in \mathcal{R}_{i}} \mu_{i}\left(C_{i} \cup\left\{s_{i}\right\}\right) \leq \tau_{i}^{\mu}\left(s_{i}\right)$

(where (iii) follows from the fact that, in equilibrium, pure strategy $s_{i}$ is necessarily used when it is the unique best reply within the consideration set at hand). Hence,

$$
\tau_{i}^{\mu}\left(r_{i}\right) \leq \sum_{C_{i} \in \mathcal{R}_{i}} \mu_{i}\left(C_{i}\right) \leq \varepsilon \cdot \sum_{C_{i} \in \mathcal{R}_{i}} \mu_{i}\left(C_{i} \cup\left\{s_{i}\right\}\right) \leq \varepsilon \cdot \tau_{i}^{\mu}\left(s_{i}\right)
$$

(where the first inequality follows from the fact that, in equilibrium, pure strategy $r_{i}$ is not used when it is not a best reply within the consideration set at hand). This establishes that $\tau^{\mu} \in M^{o}(S)$ is an $\varepsilon$-proper strategy profile in $G$. Q.E.D.

Remark 3. The above proof holds also for weaker versions of fine tenability. One such version is obtained when the hypothesis in condition (c) in Definition 7 is strengthened to also require that $D_{i}$ contains a strategy that is "strategically relevant" to the player in the sense of being a strictly better reply to some strategy profile. Formally, one may replace condition (c) by the condition (c') that if $T_{i} \neq C_{i} \subset D_{i}$ and $\max _{s_{i} \in C_{i}} u_{i}\left(\sigma_{-i},\left[s_{i}\right]\right)<\max _{s_{i} \in D_{i}} u_{i}\left(\sigma_{-i},\left[s_{i}\right]\right)$ for some $\sigma \in M(S)$, then $\mu_{i}\left(C_{i}\right) \leq \varepsilon \cdot \mu_{i}\left(D_{i}\right)$.

The following result is immediately obtained from Proposition 2, establishing the existence of at least one proper equilibrium with support in any given finely tenable block.

Corollary 1. Every finely tenable block contains the support of a proper equilibrium.

Proof: By the Bolzano-Weierstrass theorem, every sequence from a nonempty compact set has a convergent subsequence with limit in the set. Given $T$ finely tenable, let $\tau^{*}$ be such a limit point of a sequence $\left\langle\tau^{k}\right\rangle_{k \in \mathbb{N}}$ of Nash equilibria $\tau^{k} \in$ $M(F)$ of consideration-set games $G^{\mu_{k}}$ where each $\mu_{k}$ is an $\varepsilon_{k}$-proper type distribution on $T$ and $\varepsilon_{k} \rightarrow 0$. Let $\sigma^{k}$ and $\sigma^{*} \in M(S)$ be the projections of $\tau^{k}$ and $\tau^{*}$ in $G$. By construction, $\sigma^{*}$ is a proper equilibrium of $G$. Moreover, $\sigma^{*}$ has support in $T$, because $\mu^{k}(T) \rightarrow 1$ and thus $\forall s_{i} \in S_{i}$,

$$
\sigma_{i}^{k}\left(s_{i}\right)=\sum_{C_{i} \in \mathcal{C}\left(S_{i}\right)} \mu_{i}^{k}\left(C_{i}\right) \cdot \tau_{i \mid C_{i}}^{k}\left(s_{i}\right) \quad \rightarrow \quad \sigma_{i}^{*}\left(s_{i}\right)=\tau_{i \mid T_{i}}^{*}\left(s_{i}\right),
$$

so $\sigma_{i}^{*}\left(s_{i}\right)=0$ if $s_{i} \notin T_{i}$. Q.E.D. 
Remark 4. The above machinery provides a behavioral micro foundation for proper equilibrium. For by Proposition 2 as applied to $T=S$, all limit points (as $\varepsilon \rightarrow 0$ ) to projections of sequences of Nash equilibria in the associated consideration-set games are proper equilibria.

In the light of Proposition 2 and Corollary 1 it is natural to require properness when defining settledness with respect to finely tenable blocks:

Definition 9. A finely settled equilibrium is any proper equilibrium that has support in some minimal finely tenable block.

We call any equilibrium that is both finely and coarsely tenable fully settled.

Proposition 3. Every finite game has at least one fully settled equilibrium.

Proof: Let $T$ be any minimal coarsely tenable block (the existence of which follows from the finiteness of $S$ ). Then $T$ is also finely tenable. If $T$ is not a minimal finely tenable block, $T$ will contain such a block (again since $S$ is finite). According to the above corollary, there exist a proper equilibrium with support in that subblock. ${ }^{15}$ Q.E.D.

In the following elaboration of game (1) for $\alpha=\beta=\gamma=\delta=1$, the only coarsely tenable block is the whole strategy space $S$. Arguably, also this elaboration may be considered strategically inessential, so now also coarse tenability fails this invariance desideratum. By contrast, fine tenablility passes this test, and the finely settled equilibria correspond precisely to the two strict equilibria of the original game (1).

Example 2. Reconsider the extensive-form game in Example 1. If one would replace the $(0,0)$ end-node by another zero-sum subgame, like the first zero-sum subgame, the purely reduced normal form (with primed strategy labels in the second zero-sum game) would be

$\begin{array}{cccccc} & & L h^{\prime} & L t^{\prime} & R h & R t \\ \text { Game 2: } & L h & 1,1 & 1,1 & 2,-2 & -2,2 \\ & L t & 1,1 & 1,1 & -2,2 & 2,-2 \\ & R h^{\prime} & 2,-2 & -2,2 & 1,1 & 1,1 \\ & R t^{\prime} & -2,2 & 2,-2 & 1,1 & 1,1\end{array}$

\footnotetext{
${ }^{15}$ Since curb $\Rightarrow$ absorbing $\Rightarrow$ coarsely tenable $\Rightarrow$ finely tenable, every finite game in fact admits a fully settled equilibrium with support in a minimal absorbing block that is a subset of a minimal curb block.
} 
Also this elaboration of game (1) has three Nash equilibrium components:

$$
\begin{aligned}
& \left.A=\left\{\sigma=\left(p[L h]+(1-p)[L t], q\left[L h^{\prime}\right]+(1-q)\left[L t^{\prime}\right]\right)\right) \text { for } p, q \in(1 / 4,3 / 4)\right\} \\
& \left.B=\left\{\sigma=\left(p\left[R h^{\prime}\right]+(1-p)\left[R t^{\prime}\right], q[R h]+(1-q)[R t]\right)\right) \text { for } p, q \in(1 / 4,3 / 4)\right\}
\end{aligned}
$$

and $C=\left\{\sigma^{m}\right\}$, where $\sigma^{m}$ is uniform randomization over each strategy set. The proper equilibria are $\sigma^{a}=\left(\frac{1}{2}[L h]+\frac{1}{2}[L t], \frac{1}{2}\left[L h^{\prime}\right]+\frac{1}{2}\left[L t^{\prime}\right]\right) \in A, \sigma^{b}=\left(\frac{1}{2}\left[R h^{\prime}\right]+\right.$ $\left.\frac{1}{2}\left[R t^{\prime}\right], \frac{1}{2}[R h]+\frac{1}{2}[R t]\right) \in B$, and $\sigma^{m}$. The only curb, absorbing or coarsely tenable block is the whole pure-strategy space $S$, so all Nash equilibria are persistent and coarsely settled. However, $T^{a}=\{L h, L t\} \times\left\{L h^{\prime}, L t^{\prime}\right\}$ and $T^{b}=\left\{R h^{\prime}, R t^{\prime}\right\} \times\{R h, R t\}$ are finely tenable blocks by similar arguments to those given in Example 1. The game thus has only two finely, indeed fully, settled equilibria, $\sigma^{a}$ and $\sigma^{b}$, corresponding to, and behaviorally indistinguishable from, the two strict equilibria of the original game (1). In other words, fine tenability treats each zero-sum subgame here as strategically equivalent to its value.

Examples 1 and 2 show that neither persistence, curb nor coarse tenability is invariant in the purely reduced normal form under replacement of an end-node in an extensive-form game by a subgame that has a unique Nash equilibrium, where this equilibrium is totally mixed and results in the same payoff vector as the end node. ${ }^{16}$ By contrast, fine tenability exhibits such invariance in these two examples, and we proceed to show that this is a general property of fine tenability. Suppose that $T$ is a block in any given finite game $G=\langle N, S, u\rangle$, and let $r \in S$ be any pure-strategy profile in the game. Let us construct a new game $\hat{G}$ that differs from the given game in that, for each player $i$, the strategy $r_{i}$ is replaced by a nonempty set $R_{i}$ of new pure strategies. The payoffs $\hat{u}$ in $\hat{G}$ are all the same as in $G$, with each new strategy in $R_{i}$ being payoff equivalent to $r_{i}$ in $G$ except when all players use new strategies in the block $R=\times_{i \in I} R_{i}$. Let $\hat{T}$ be the block in the new game which corresponds to the block $T$ in the given game. That is, each set $\hat{T}_{i}$ is the same as $T_{i}$ except that, if $r_{i} \in T_{i}$ then $\hat{T}_{i}$ includes all strategies in $R_{i}$, instead of $r_{i}$, and if $r_{i} \notin T_{i}$ then $\hat{T}_{i}$ includes no strategy in $R_{i}$. Suppose that the block game $\hat{G}_{R}=\langle N, R, \hat{u}\rangle$ in $\hat{G}$ has a unique Nash equilibrium, and suppose this is totally mixed (in the block game) and results in payoffs $u(r) \in \mathbb{R}^{n}$.

Proposition 4. If the block $T$ is finely tenable in $G$, then the corresponding block $\hat{T}$ is finely tenable in the game $\hat{G}$ as constructed above.

\footnotetext{
${ }^{16}$ See Mailath, Samuelson and Swinkels (1993) for an analysis of how subgames and information sets in a finite extensive-form game are represented in its purely reduced normal form.
} 
Proof: For the new game $\hat{G}$, consider a sequence of $\varepsilon$-proper type distributions with respect to $\hat{T}$ and Nash equilibria on the corresponding random considerationset games, where $\varepsilon \rightarrow 0$ in the sequence. Choosing a subsequence if necessary, we can assume that each player's preference ordering over his pure strategies, given the other players' behavior in the random consideration-set equilibria, is constant over the sequence. (There are only finitely many ways that each player can weakly order his pure strategies in $\hat{G}$, and so at least one ordering must be repeated infinitely.) By compactness we can also assume (again choosing a subsequence if necessary) that, for each player $i$, the conditional probability distribution for $i$ 's mixed strategy, given that $i$ 's choice is in $R_{i}$, converges to some limiting conditional mixed strategy in $\Delta\left(R_{i}\right)$.

For each player $i$, either all or none of $R_{i}$ is in $\hat{T}_{i}$. In either case, conditional on the event that $i$ has a best considered response in $R_{i}$ (which happens when $i$ considers something in $R_{i}$ but nothing in the constant set of alternatives that he would prefer to every strategy in $R_{i}$ ), the probability of $i$ considering all of $R_{i}$ goes to 1 as $\varepsilon \rightarrow 0$. Player $i$ 's choice among his new strategies in $R_{i}$ depends only on the event that the others are all in the new block $R$ (an event which has positive probability in the random consideration-set game), because otherwise these new strategies are, by construction, payoff equivalent for him. So in the limit, the conditional probability distribution for every player's mixed strategy, given that the choice is in the set of new strategies, must converge to the unique equilibrium of the block game $\hat{G}_{R}$ on $R$ (as in the limit each player $i$ puts conditional probability 1 on his set of best responses in $R_{i}$ ). This equilibrium is totally mixed, and so all player $i$ 's pure strategies in $R_{i}$ must all give him the same expected utility also in the random consideration-set equilibria, because otherwise the probability of the worst alternative in $R_{i}$ would have probability 0 in the limit of $i$ 's conditional mixed strategy in $\Delta\left(R_{i}\right)$. But every player $i$ can be indifferent among all of his new strategies in $R_{i}$ only if they are all playing the unique equilibrium on the $R$ block. Thus, conditional on the event that all other players are in the block $R$, any strategy in $R_{i}$ would give player $i$ an expected payoff equal to $u_{i}(r)$, and in any other event each new strategy in $R_{i}$ also yields the same payoffs as strategy $r_{i}$ in the given game.

Now these $\varepsilon$-proper-on- $\hat{T}$ random consideration-set perturbations of $\hat{G}$ induce corresponding random consideration-set perturbations of the original game $G$ with respect to $T$. Here we let the consideration or choice of any strategy in $R_{i}$ in $\hat{G}$ correspond to the consideration or choice of $r_{i}$ in $G$, and of course any other pure strategy corresponds to itself identically across $G$ and $\hat{G}$. (That is, the event in $G$ of player $i$ considering any set of pure strategies corresponds to the event in $\hat{G}$ of $i$ considering the same set if the set excludes $r_{i}$, and otherwise it corresponds to the event in $\hat{G}$ of $i$ considering the same elements outside of $R_{i}$ plus at least one element in $R_{i}$.) 
It can be verified that $\varepsilon$-properness on $\hat{T}$ for a type distribution $\hat{\mu}$ in $\hat{G}$ implies that the corresponding type distribution $\mu$ in $G$ is $\varepsilon$-proper on $T$. To see this, let $\hat{\mu}_{i}$ be the given $\varepsilon$-proper-on- $\hat{T}_{i}$ type distribution for a player $i$ in the perturbed random consideration-set game on $\hat{G}$, and let $\mu_{i}$ be the corresponding distribution for the perturbed random consideration-set game on $G$. We need to show that, if $T_{i} \neq C_{i} \subset$ $D_{i} \subseteq S_{i}$ then $\mu_{i}\left(C_{i}\right) \leq \varepsilon \mu_{i}\left(D_{i}\right)$. If $r_{i} \notin D_{i}$ then this holds because

$$
\mu_{i}\left(C_{i}\right)=\hat{\mu}_{i}\left(C_{i}\right) \leq \varepsilon \hat{\mu}_{i}\left(D_{i}\right)=\varepsilon \mu_{i}\left(D_{i}\right) .
$$

If $r_{i} \in C_{i}$, then $r_{i} \in D_{i}$ and so

$$
\mu_{i}\left(C_{i}\right)=\sum_{Z_{i} \in \mathcal{C}\left(R_{i}\right)} \hat{\mu}_{i}\left(Z_{i} \cup C_{i} \backslash r_{i}\right) \leq \sum_{Z_{i} \in \mathcal{C}\left(R_{i}\right)} \varepsilon \hat{\mu}_{i}\left(Z_{i} \cup D_{i} \backslash r_{i}\right)=\varepsilon \mu_{i}\left(D_{i}\right) .
$$

(As before, $\mathcal{C}\left(R_{i}\right)$ denotes the set of nonempty subsets of $\left.R_{i}\right)$. Finally, if $r_{i} \notin C_{i}$ but $r_{i} \in D_{i}$ then $C_{i}$ is a subset of $Z_{i} \cup D_{i} \backslash r_{i}$ for any $Z_{i} \in \mathcal{C}\left(R_{i}\right)$, and so we get

$$
\mu_{i}\left(C_{i}\right)=\hat{\mu}_{i}\left(C_{i}\right) \leq \sum_{Z_{i} \in \mathcal{C}\left(R_{i}\right)} \varepsilon \hat{\mu}_{i}\left(Z_{i} \cup D_{i} \backslash r_{i}\right)=\varepsilon \mu_{i}\left(D_{i}\right) .
$$

Of course if $C_{i}=T_{i}$ then we get $\mu_{i}\left(C_{i}\right)=\mu_{i}\left(T_{i}\right) \geq \hat{\mu}_{i}\left(\hat{T}_{i}\right) \geq 1-\varepsilon$.

For any player $i$ and any consideration set $C_{i}$ in $G$, for all consideration sets in $\hat{G}$ that correspond to $C_{i}, i$ 's equilibrium best responses in our random consideration-set perturbations of $\hat{G}$ all correspond to the same pure strategies in $C_{i}$. So the corresponding random consideration-set perturbation of $G$ has a corresponding equilibrium where the probability in $G$ of any player $i$ choosing any pure strategy in any consideration set is the conditional probability in $\hat{G}$ of player $i$ choosing a corresponding pure strategy given that player $i$ is considering one of the corresponding consideration sets in $\hat{G}$. Corresponding pure strategies for player $i$ give him the same expected payoffs in both corresponding equilibria. But the block $T$ is finely tenable in $G$. So when $\varepsilon>0$ is small enough, the pure strategies in $T_{i}$ must be global best responses in equilibrium for each player $i$. Hence, the corresponding choices in $\hat{T}_{i}$ must also be global best responses in equilibrium when $\varepsilon$ is small enough. Thus, $\hat{T}$ is finely tenable in $\hat{G}$. Q.E.D.

The finely settled equilibria in the Example 2 are also coarsely settled. The next example shows that is not always the case.

Example 3. Consider a version of the battle-of-the-sexes game where player 1 has an outside option: 


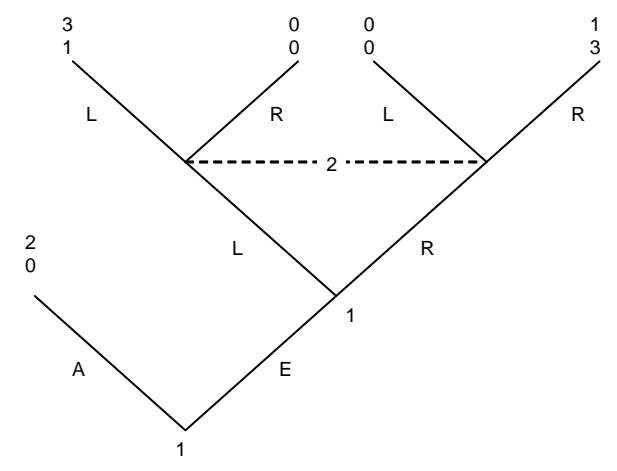

Its purely reduced normal form (with $A$ representing the two payoff-equivalent strategies $A L$ and $A R$ ) is

$$
\begin{array}{cccc} 
& & L & R \\
\text { Game 3: } & E L & 3,1 & 0,0 \\
& E R & 0,0 & 1,3 \\
& A & 2,0 & 2,0
\end{array}
$$

This normal-form game has two Nash equilibrium components, the singleton set $T^{*}=$ $\{E L\} \times\{L\}$, consisting of the strict pure equilibrium $s^{*}=(E L, L)$ usually referred to as the "forward-induction" solution (see Kohlberg and Mertens, 1986, and van Damme, 1989), and a continuum component in which player 1 plays $A$ for sure while player 2 plays $R$ with probability at least $1 / 3$. The strict equilibrium $s^{*}$ is fully settled. Another proper equilibrium of this game is $s^{o}=(A, R)$, corresponding to the sequential equilibrium of the extensive-form game in which play of $(R, R)$ is expected in the battle-of-sexes subgame. (To see that $s^{o}$ is proper, note that for all $\varepsilon>0$ small enough, $\sigma_{1}^{\varepsilon}=\left(\varepsilon^{2}, \varepsilon, 1-\varepsilon-\varepsilon^{2}\right)$ and $\sigma_{2}^{\varepsilon}=(\varepsilon, 1-\varepsilon)$ make up an $\varepsilon$-proper strategy profile $\sigma^{\varepsilon}$.) Clearly its supporting block $T^{o}=\{A\} \times\{R\}$ is not coarsely tenable, since strategy $L$ is the unique best reply if there is a positive probability that 1 plays $E L$, which indeed is the case under type distributions that attach arbitrary little, but positive probability to $\{E L\}$ being 1's consideration set, and zero probability to all other non-conventional consideration sets (that is, other than $T_{1}^{o}$ ). Nevertheless, $T^{o}$ is finely tenable. To see this, let $\mu$ be an $\varepsilon$-proper type distribution on $T^{o}$ and let $\tau \in M(F)$ be any Nash equilibrium of $G^{\mu}$. Then $\tau_{2}^{\mu}(L)<\varepsilon$, so for $\varepsilon>0$ small enough $\tau_{1}^{\mu}(E L) \leq \mu_{1}(\{E L\}) \leq \varepsilon \cdot \mu_{1}(\{E L, E R\}) \leq \tau_{1}^{\mu}(E R)$, which implies that $A$ and $R$ are best replies to $\tau^{\mu}$. In sum, while $s^{*}$ is fully settled, $s^{o}$ is finely but not coarsely settled.

While coarse tenability requires robustness to "all" type distributions (that assign much probability to the conventional types), fine tenability only requires robustness to such "rationality biased" type distributions. So why does this emphasis on rational types then not imply forward induction? It is true that player 1 would never use the 
strictly dominated strategy $E R$ when she considers her whole strategy set, but that does not make $E R$ less likely than $E L$, because she would not use either when she considers the whole set $S_{1}$ if player 2 is much more likely to use $R$ than $L$. The block $T^{o}$, while not coarsely tenable, is supported as finely tenable because when player 2 is much more likely to consider only $R$, then player 1's strict preference is $A \succ E R \succ E L$. Hence, when she accidentally considers only $\{E R, E L\}$, which by fine tenability is the most likely way that she will not do $A$, she will do $E R$. So $E R$ is much more likely than EL, and so 2 indeed strictly prefers $R$ over $L .^{17}$

We proceed to establish that coarsely and finely tenable blocks, and thus also coarsely and finely settled equilibria, are generically equivalent.

\section{Generic normal-Form Games}

The concept of regular equilibrium was introduced by Harsanyi (1973) and slightly modified by van Damme (1991), who defined a Nash equilibrium of a finite normalform game to be regular if the Jacobian, associated with a certain system of equations closely related to those characterizing Nash equilibrium, is non-singular (op. cit. Definition 2.5.1).

Definition 10. A game $G=\langle N, S, u\rangle$ is hyper-regular if, for every block $T \subseteq S$, all Nash equilibria of the associated block game $G_{T}$ are regular in the sense of van Damme (1991).

In a well-defined sense, almost all normal-form games are hyper-regular:

Lemma 1. For any (finite) set of players $N$ and (finite) sets of strategies $S_{i}$ for each player $i \in N$, the set of payoff functions $u \in \mathbb{R}^{|N| \cdot|S|}$ such that $G=\langle N, S, u\rangle$ is not hyper-regular is contained in a closed set of Lebesgue measure zero.

Proof: The property of regularity of a block game $G_{T}$ depends only on the payoffs on $T$, and this property will fail only for payoff functions in a closed set of Lebesgue measure zero (van Damme, 1991, Theorem 2.6.1). There are only finitely many blocks $T \subseteq S$, and the union of finitely many such sets is still a closed set of measure zero. Q.E.D.

Proposition 5. If a game $G=\langle N, S, u\rangle$ is hyper-regular, then any block $T$ is finely tenable if and only if it is coarsely tenable. Any equilibrium of $G$ is finely settled if and only if it is coarsely settled.

\footnotetext{
${ }^{17}$ See also Battigalli and Siniscalchi (2002) and Battigalli and Friedenberg (2012) for epistemic analyses of forward-induction reasoning.
} 
Proof: We first establish that for a hyper-regular game there cannot exist any Nash equilibrium $\tau$ of any block game $G_{T}$ such that a player $i$ has an alternative $s_{i} \in S_{i} \backslash T_{i}$ with $u_{i}\left(\tau_{-i},\left[s_{i}\right]\right)=u_{i}(\tau)$. If this equality would hold, and if we added $s_{i}$ to $T_{i}$ (obtaining $T_{i}^{\prime}=T_{i} \cup\left\{s_{i}\right\}$ ), then we would obtain a block game $G_{T^{\prime}}$ in which $\tau$ would still be a Nash equilibrium but, having an alternative best reply in $T^{\prime}$ that gets zero probability in $\tau, \tau$ would not be quasi-strict in this new block game $G_{T^{\prime}}$. By hyper-regularity of $G, \tau$ is a regular equilibrium of $G_{T^{\prime}}$ and hence $\tau$ is quasi-strict (Corollary 2.5.3 in van Damme, 1991), a contradiction. ${ }^{18}$ So if $\tau$ is a Nash equilibrium of some block game $G_{T}$, then either $u_{i}\left(\tau_{-i},\left[s_{i}\right]\right)>u_{i}(\tau)$ or $u_{i}\left(\tau_{-i},\left[s_{i}\right]\right)<u_{i}(\tau)$. The first of these inequalities, for any $i$ and $s_{i}$, would imply that $T$ is not coarsely tenable. The second inequality, for all $i$ and $s_{i}$, would imply that $T$ is coarsely tenable. Thus, in the given hyper-regular game $G$, a block $T$ is coarsely tenable if and only if $u_{i}\left(\tau_{-i},\left[s_{i}\right]\right)<u_{i}(\tau)$ for all $i$ and $s_{i} \in S_{i} \backslash T_{i}$, at all equilibria $\tau$ of the block game. Coarsely tenable blocks are always finely tenable, so it remains to prove that, for our hyper-regular game $G$, any block $T$ that is not coarsely tenable is not finely tenable.

In order to establish this, consider any Nash equilibrium $\tau$ of any block game $G_{T}$. The payoff function of $G_{T}$ can be viewed as a vector $u$ in $\mathbb{R}^{|N| \cdot|T|}$. By hyperregularity of $G$, the equilibrium $\tau$ is regular, and thus also strongly stable, in $G_{T}$ (van Damme, 1991, Definition 2.4.4 and Theorem 2.5.5). This means that there is some open neighborhood $V$ of $\tau \in M(T)$ and some open neighborhood $U$ of $u \in \mathbb{R}^{|N| \cdot|T|}$ such that, for any perturbation of $G_{T}$ that has a payoff function $\tilde{u}$ in $U$, we obtain a game $\tilde{G}_{T}=\langle N, T, \tilde{u}\rangle$ that has exactly one equilibrium $\tilde{\tau}$ in $V$, and this equilibrium depends continuously on the payoff function $\tilde{u}$. Now let's think about a considerationset game $G^{\mu}$ and a block $T$. For each player $i$ let $\rho_{i}: \mathcal{C}\left(S_{i}\right) \backslash T_{i} \rightarrow \Delta\left(S_{i}\right)$ define a mixed strategy $\rho_{i \mid C_{i}} \in \Delta\left(C_{i}\right)$ for every consideration set $C_{i}$ other than $T_{i}$. Let $B(T)$ be the set of all such function profiles $\rho=\left(\rho_{1}, . ., \rho_{n}\right)$. Then $\rho$ defines the behavior of players at all consideration sets other than those of $T$, and the only question remaining in $G^{\mu}$ is what a player $i$ would do when considering $T_{i}$, which will happen with probability at least $1-\varepsilon$. So with any given $\varepsilon$, the consideration-set game $G^{\mu}$ becomes a perturbation of $G_{T}$, and its payoff function in $\mathbb{R}^{|N| \cdot|T|}$ will be in the open set $U$ for all $\varepsilon>0$ sufficiently small, given $\rho$. In fact, there exists an $\bar{\varepsilon}>0$ such that $\tilde{u} \in U$ for all $\rho$. Now, given any $\varepsilon \in(0, \bar{\varepsilon})$, consider the correspondence that sends any profile $\rho \in B(T)$ to each player $i$ 's (non-empty, compact and convex) best replies at every consideration set $C_{i} \neq T_{i}$ to the $\rho$ and $\tilde{\tau}$ strategies, where $\tilde{\tau}$ is the (continuously defined) equilibrium in $V$ for this perturbation of $G_{T}$. This correspondence is upper hemi-continuous in $\rho$, so, by Kakutani's fixed-point theorem, for any such $\varepsilon$, there

\footnotetext{
${ }^{18}$ A quasi-strict equilibrium (Harsanyi, 1973) is any Nash equilibrium in which all players use all their pure best replies.
} 
exists a fixed point $\rho^{*}$. This fixed-point $\rho^{*}$, together with its corresponding $\tilde{\tau}$ at $T$, will constitute an equilibrium of the consideration-set game $G^{\mu}$. The projection of this equilibrium to $M(S)$ will be $\varepsilon$-proper with respect to the block $T$ (along the lines given in Section 5) and these strategy profiles converge to the given block-game equilibrium $\tau$ as $\varepsilon \rightarrow 0$. But then this sequence would yield a contradiction of $T$ being tenable if we had $u_{i}\left(\tau_{-i},\left[s_{i}\right]\right)>u_{i}(\tau)$ for some player $i$ and some strategy $s_{i} \in S_{i} \backslash T_{i}$. Thus, if $T$ is not coarsely tenable, then $T$ is not finely tenable either. So for a hyper-regular game, a block is coarsely tenable if and only if it is finely tenable.

Since all regular equilibria are proper (van Damme, 1991, Theorems 2.5.5, 2.4.7, 2.3.8), an equilibrium in a hyper-regular game is coarsely settled if and only if it is finely settled. Q.E.D.

The game in the introduction shows that there are generic games in which the set of settled equilibria is strictly smaller than the set of Nash (perfect, proper) equilibria. The game in the next example, taken from Table 7 in Myerson (1996), has an open neighborhood (in the space of $2 \times 4$ normal-form games) in which there is always a Nash equilibrium which is persistent but not settled. This shows that settled equilibrium is not generically equivalent with Nash or persistent equilibrium.

Example 4. Consider the game

$$
\begin{aligned}
& a_{2} \quad b_{2} \quad c_{2} \quad d_{2} \\
& \text { Game 4: } \quad a_{1} \quad 0,2 \quad 1,1 \quad 0,0 \quad 1,-3 \\
& b_{1} \quad 1,-3 \quad 0,0 \quad 1,1 \quad 0,2
\end{aligned}
$$

and note that the block game over the "middle" block $T^{b c}=\left\{a_{1}, b_{1}\right\} \times\left\{b_{2}, c_{2}\right\}$ is identical with game (1) for $\alpha=\beta=\gamma=\delta=1$. The diagram below shows the payoffs to player 2's pure strategies as functions of the probability $p$ by which player 1 uses her first pure strategy.
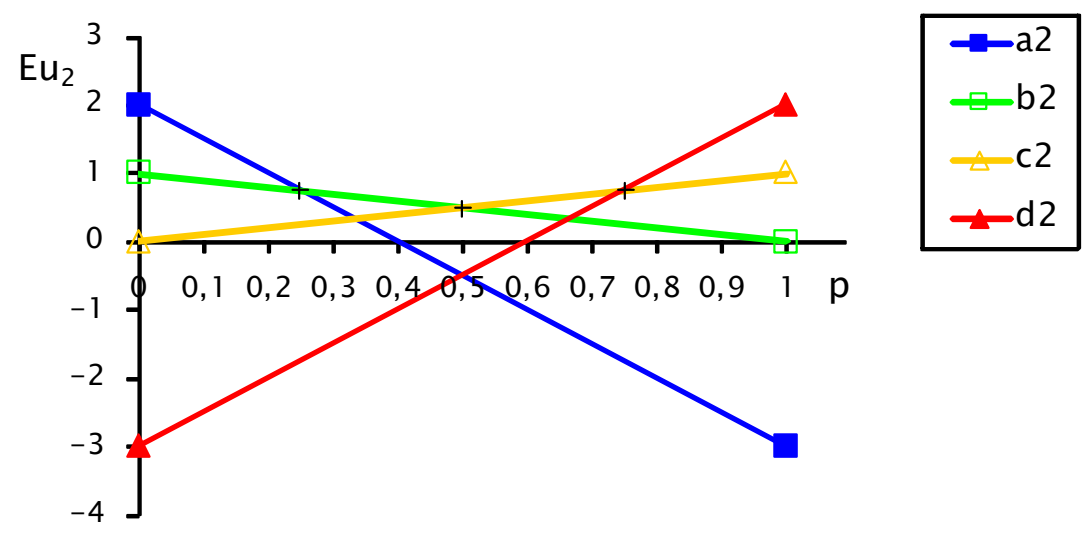
This game has three Nash equilibria, all mixed. In each equilibrium, player 1 uses both her pure strategies while player 2 uses only two of his four pure strategies; either the two left-most, $\left\{a_{2}, b_{2}\right\}$, the two middle ones, $\left\{b_{2}, c_{2}\right\}$, or the two rightmost, $\left\{c_{2}, d_{2}\right\}$, each equilibrium corresponding to a kink in the upper envelope of the payoff lines in the above diagram.

The game is hyper-regular. This follows from Theorem 7.4 in Jansen (1981) (see also Theorem 3.4.5 in van Damme, 1991), according to which a Nash equilibrium of a finite two-player game is regular if and only if it is essential and quasi-strict. ${ }^{19}$ It is not difficult to verify that all block equilibria, of all blocks in this game, have both properties. Moreover, the game has only one curb block, the whole set $S$, and it has only one absorbing retract, the whole set $M(S)$. Hence, all three equilibria are persistent. However, the "middle" equilibrium is not settled.

More exactly, the three Nash equilibria of this game are

$$
\begin{aligned}
\sigma^{a b} & =\left(\frac{3}{4}\left[a_{1}\right]+\frac{1}{4}\left[b_{1}\right], \frac{1}{2}\left[a_{2}\right]+\frac{1}{2}\left[b_{2}\right]\right) \\
\sigma^{b c} & =\left(\frac{1}{2}\left[a_{1}\right]+\frac{1}{2}\left[b_{1}\right], \frac{1}{2}\left[b_{2}\right]+\frac{1}{2}\left[c_{2}\right]\right) \\
\sigma^{c d} & =\left(\frac{1}{4}\left[a_{1}\right]+\frac{3}{4}\left[b_{1}\right], \frac{1}{2}\left[c_{2}\right]+\frac{1}{2}\left[d_{2}\right]\right)
\end{aligned}
$$

Consider first the "middle" block $T^{b c}=\left\{a_{1}, b_{1}\right\} \times\left\{b_{2}, c_{2}\right\}$, the support of $\sigma^{b c}$. The associated block contains, in addition to $\sigma^{b c}$, two (strict pure) block-game equilibria, each, however, with better replies outside the block. Hence, by Proposition 1, this block is not coarsely tenable. Arguably, if $T^{b c}$ became the conventional block played in a population, play might drift towards one of these strict block equilibria, which would induce a movement out of the block, towards a better reply, and thereby destabilize the block. By contrast, the supports of each of the two other equilibria, the "side" blocks $T^{a b}=\left\{a_{1}, b_{1}\right\} \times\left\{a_{2}, b_{2}\right\}$ and $T^{c d}=\left\{a_{1}, b_{1}\right\} \times\left\{c_{2}, d_{2}\right\}$, do not contain any other block equilibria and are coarsely tenable. The only coarsely tenable block that contains $\sigma^{b c}$ is $S$, which, however, is not minimal. Hence, while all three equilibria are persistent, only $\sigma^{a b}$ and $\sigma^{c d}$ are coarsely settled. These claims hold for an open set of payoff perturbations of the game. Thus, the property of being coarsely settled is not generically equivalent to persistence.

The two minimal coarsely tenable blocks, $T^{a b}$ and $T^{c d}$, are, a fortiori, also finely tenable. Since they contain no other finely tenable block, they are minimal and hence

\footnotetext{
${ }^{19}$ An essential equilibrium (Wu and Jiang, 1962) is any Nash equilibrium such that every nearby game, in terms of payoffs, has some nearby Nash equilibrium.
} 
$\sigma^{a b}$ and $\sigma^{c d}$ are also finely settled. Is the middle block $T^{b c}$ finely tenable? As we will see, this is not the case although $T^{b c}$ is the support of a proper equilibrium, $\sigma^{b c}$. First, to see that $\sigma^{b c}$ is proper, let $\sigma_{1}^{\varepsilon}=(1 / 2,1 / 2)$ and $\sigma_{2}^{\varepsilon}=(\varepsilon, 1 / 2-\varepsilon, 1 / 2-\varepsilon, \varepsilon)$. Clearly $\sigma^{\varepsilon}$ is $\varepsilon$-proper for all $\varepsilon \in(0,1 / 2)$, and $\sigma^{\varepsilon} \rightarrow \sigma^{b c}$ as $\varepsilon \rightarrow 0$. Second, to see that $T^{b c}$ is not finely tenable, let $\varepsilon>0$ and let $\mu$ be as in Remark 2 , with $\delta_{2}\left(b_{2}\right)=\varepsilon$, $\delta_{2}\left(d_{2}\right)=\varepsilon \cdot \delta_{2}\left(c_{2}\right)=\varepsilon^{2} \cdot \delta_{2}\left(a_{2}\right)$ and $\left\|\delta_{i}\right\|<\varepsilon /(1+\varepsilon)$ for both players $i$. Then $\mu$ is $\varepsilon$-proper on $T^{b c}$ for all $\varepsilon>0$. However, for all $\varepsilon>0$ sufficiently small and all Nash equilibria $\tau \in M(F)$ of $G^{\mu}$ we have

$$
u_{2}\left(\tau_{1}^{\mu}, \tau_{2 \mid T_{2}}\right)<u_{2}\left(\tau_{1}^{\mu},\left[a_{2}\right]\right),
$$

so $T^{b c}$ is not finely tenable. In sum: $\sigma^{a b}$ and $\sigma^{c d}$ are the only fully settled equilibria of this game.

\section{More EXAMPles}

Suppose that game (1) was enlarged by letting each player's mixed Nash-equilibrium strategy be represented as a new pure strategy. As the following example shows, this would not affect the collection of minimal tenable blocks.

Example 5. Let $\alpha=\beta=\gamma=\delta=1$, and consider

\begin{tabular}{|c|c|c|c|c|}
\hline \multirow{3}{*}{ Game 5: } & & $a_{2}$ & $b_{2}$ & $c_{2}$ \\
\hline & $a_{1}$ & 1,1 & 0,0 & $\lambda, \lambda$ \\
\hline & $b_{1}$ & 0,0 & 1,1 & $\lambda, \lambda$ \\
\hline & $c_{1}$ & $\lambda, \lambda$ & $\lambda, \lambda$ & $\lambda, \lambda$ \\
\hline
\end{tabular}

for $\lambda<1$. For $\lambda=1 / 2$, the new pure strategy $c_{i}$ is payoff equivalent with the mixed Nash equilibrium strategy $\sigma_{i}^{*}=\frac{1}{2}\left[a_{i}\right]+\frac{1}{2}\left[b_{i}\right]$, for $i=1,2$, in game (1). For $\lambda=1 / 2$, this $3 \times 3$-game has infinitely many Nash equilibria; the two strict equilibria $\left(a_{1}, a_{2}\right)$ and $\left(b_{1}, b_{2}\right)$, the pure equilibrium $\left(c_{1}, c_{2}\right)$, and a continuum of mixed equilibria where each player $i$ randomizes arbitrarily between $\sigma_{i}^{*}$ and $c_{i}$ (thus also including $\left(c_{1}, c_{2}\right)$ as an extreme point). The two strict equilibria are of course fully settled. Moreover, their supports are the only minimal tenable blocks (and this holds for an open set of payoffs around $\alpha=\beta=\gamma=\delta=1$ ). In particular, the singleton block $T^{c}=\left\{c_{1}\right\} \times\left\{c_{2}\right\}$ is not coarsely tenable, and this holds for all $\lambda<1$. The reason is that for arbitrarily small $\varepsilon>0$ there are type distributions $\mu$ such that $\mu_{i}\left(\left\{c_{i}\right\}\right)>1-\varepsilon$ for $i=1,2$, under which $c_{i}$ is not a best reply to the projection of any Nash equilibrium in the associated consideration-set game $G^{\mu}$. For example, for each player $i$ let $\mu_{i}\left(\left\{c_{i}\right\}\right)=1-\varepsilon / 2$ and $\mu_{i}\left(\left\{a_{i}\right\}\right)=\varepsilon / 2$ (thus all other consideration sets have probability zero). Let $\tau$ be any Nash equilibrium of $G^{\mu}$. Then $\tau_{i}^{\mu}\left(a_{i}\right)=\varepsilon / 2$ and $\tau_{i}^{\mu}\left(b_{i}\right)=0$, so $\max _{t_{i} \in T_{i}^{c}} u_{i}\left(\tau_{-i}^{\mu},\left[t_{i}\right]\right)=\lambda$ while (for any $\lambda<1$ )

$$
\max _{s_{i} \in S_{i}} u_{i}\left(\tau_{-i}^{\mu},\left[s_{i}\right]\right)=1 \cdot \varepsilon / 2+\lambda \cdot(1-\varepsilon / 2)>\lambda .
$$


Hence, our solution concepts reject the mixed equilibrium in these games even when this is rendered "minimal" by way of representing it as a pair of pure strategies. Its rejection is thus not due to the requirement of block minimality.

Established refinements, such as Kohlberg-Mertens stability, are known to have little "bite" in sender-receiver games. By contrast, settledness effectively discards arguably implausible equilibria in such games. We illustrate this by way of a simple example due to Balkenborg, Hofbauer and Kuzmics (2014). ${ }^{20}$

Example 6. Consider a sender-receiver game in which there are two equally likely states of nature, $\omega=A$ and $\omega=B$. Player 1, the sender, observes the state of nature and sends one of two messages, $a$ or $b$, to player 2. Having received 1's message, 2 takes one of two actions, $\alpha$ or $\beta$. Hence, each player has four pure strategies. Assume that both players receive payoff 2 if action $\alpha(\beta)$ is taken in state $A(B)$, and otherwise both players receive payoff zero. The normal form of this game is

$\begin{array}{llllll} & & \alpha \alpha & \alpha \beta & \beta \alpha & \beta \beta \\ \text { Game 6: } & a a & 1,1 & 1,1 & 1,1 & 1,1 \\ & a b & 1,1 & 2,2 & 0,0 & 1,1 \\ & b a & 1,1 & 0,0 & 2,2 & 1,1 \\ & b b & 1,1 & 1,1 & 1,1 & 1,1\end{array}$

Any strategy pair that assigns equal probability to the two middle strategies is a Nash equilibrium: if $\sigma_{1}=(p, q, q, r) \in \Delta\left(S_{1}\right)$ and $\sigma_{2}=\left(p^{\prime}, q^{\prime}, q^{\prime}, r^{\prime}\right) \in \Delta\left(S_{2}\right)$, then $\sigma$ is a Nash equilibrium. As pointed out by Balkenborg et al. (2014), every Nash equilibrium $\sigma$ of this kind, viewed as a singleton set, is strategically stable in the sense of Kohlberg and Mertens (1986). In these equilibria the players have not established a convention in their communication; player 2 does not know if a "means" $\alpha$ and $b$ "means" $\beta$, or if it is the other way round. By contrast, in each of the two pure and strict equilibria, $s^{*}=(a b, \alpha \beta)$ and $s^{* *}=(b a, \alpha \beta)$, it is as if the players have established a convention in their communication. For the same reasons as given in the preceding example, only these two equilibria are settled.

Also voting games are well-known to exhibit a plethora of Nash equilibria, many of which seem unreasonable and yet resist standard refinements such as perfection and strategic stability. The next example suggests that the present solutions have good cutting power, beyond that of perfection and strategic stability, and that it selects natural equilibria. The example concerns the so-called Duverger's law, which asserts that the plurality rule for selecting the winner of elections favors the two-party (or two-candidate) system, see Riker (1982).

\footnotetext{
${ }^{20}$ See also the analysis of related issues in Gordon (2011).
} 
Example 7. There are three candidates, call them 1, 2, and 3, of whom candidates 1 and 2 are ideologically similar, say on the left of the political spectrum, and then candidate 3 is on the right side of the spectrum. In the election, each voter must choose to vote for one candidate, and the candidate with the most votes wins; a tie for the most would be resolved by random selection among those in the tie. There are seven voters. Each voter can be characterized by his or her vector $v=\left(v_{1}, v_{2}, v_{3}\right)$ of utilities for each of the three candidates winning the election. Three voters are rightist partisans of candidate 3 and have utility vector $(0,0,1)$. The other four voters are leftists and can be called $1 A$ with utility vector $(4,1,0), 1 B$ with utility vector $(3,2,0), 2 A$ with utility vector $(1,4,0)$, and $2 B$ with utility vector $(2,3,0)$. In any proper equilibrium, all three rightists always vote for the rightist candidate 3.

We find two settled equilibria that have two candidates getting all the votes, as predicted by Duverger's law for such plurality elections. In one of these settled equilibria, all four leftist voters, $\{1 A, 1 B, 2 A, 2 B\}$, vote for candidate 1 , who then wins the election with probability 1 . In the other of these settled equilibria, all four leftist voters vote for candidate 2 , who then wins. In either of these equilibria, if one leftist voter deviated to vote for the other leftist candidate, there would be a tie, and the rightist candidate would win with probability 1/2. Each of these Duvergerian equilibria is a strict pure equilibrium, and its support is a minimal tenable block.

But there is also a third equilibrium, one in which the leftist voters split their votes among candidates 1 and 2 . In this equilibrium, voter $1 A$ votes for candidate 1 for sure, voter $1 B$ randomizes, voting for candidate 1 with probability 0.6 and for candidate 2 with probability 0.4 , voter $2 A$ votes for candidate 2 for sure, and voter $2 B$ randomizes, voting for candidate 2 with probability 0.6 and for candidate 1 with probability 0.4. In this equilibrium, the rightist candidate 3 wins with probability 0.76 while each of the leftist candidates has probability 0.12 of winning. This equilibrium violates Duverger's law, and it is not settled. The support of this equilibrium is not tenable, because its block game would also have pure-strategy equilibria in which voters $1 B$ and $2 B$ both vote for the same leftist candidate (candidate 1 or 2 ), which would not be an equilibrium of the original game because it would make voter $1 \mathrm{~A}$ or $2 A$ switch over to also vote for the same leftist candidate. Thus, any tenable block that includes the support of this mixed equilibrium must also include the support of both Duvergerian equilibria, and so it is not minimal among all tenable blocks. Arguably, in rejecting this mixed equilibrium (despite the fact that it is regular), settledness formalizes the intuition that it does not appear as a likely convention if this or similar elections were held in a familiar context. Presumably, left-wing voters would then come to "agree" to support one of the two left-wing candidates.

Our final example shows that, unlike minimal curb blocks, minimal tenable blocks may overlap. It also provides yet another illustration of how settledness can be strictly 
more selective than persistence.

Example 8. Consider

$\begin{array}{ccccc} & & a_{2} & b_{2} & c_{2} \\ \text { Game 8: } & a_{1} & 3,1 & 1,3 & 0,0 \\ & b_{1} & 1,3 & 3,1 & 1,3 \\ & c_{1} & 0,0 & 1,3 & 3,1\end{array}$

This game has three Nash equilibria: $\sigma^{a b}$, in which each player randomizes uniformly across his or her two first pure strategies, $\sigma^{b c}$, in which they randomize uniformly across their last two pure strategies, and the totally mixed strategy profile

$$
\sigma^{m}=\left(\frac{2}{9}\left[a_{1}\right]+\frac{5}{9}\left[b_{1}\right]+\frac{2}{9}\left[c_{1}\right], \frac{2}{5}\left[a_{2}\right]+\frac{1}{5}\left[b_{2}\right]+\frac{2}{5}\left[c_{2}\right]\right) .
$$

The supports of $\sigma^{a b}$ and $\sigma^{b c}$ are blocks that both contain the strategy profile $\left(b_{1}, b_{2}\right)$. These blocks are not absorbing, since for certain mixed-strategy profiles near the profile that puts unit probability on $b_{1}$ and $b_{2}$, either player 1 or 2 has no best reply in the block. However, they are minimal coarsely tenable. The only absorbing block is the full strategy space $S$. All Nash equilibria are persistent, while only $\sigma^{a b}$ and $\sigma^{b c}$ are coarsely, indeed, fully settled.

\section{Conclusion}

This paper has focused on an assumption that, in culturally familiar games, people will develop social conventions that simplify the game by excluding some strategies from normal consideration. We feel that such an assumption has substantial realism. Our assumption, to allow for the possibility that a player may ignore some strategies that are feasible in the actual game, leads us to analyze consideration-set games where the strategies that each player conventionally considers may be a subset of those actually feasible. But we allow players to break free from such conventions and explore other strategies in the game. Thus, our concepts of tenable strategy blocks have been defined as conditions for a conventional simplification to justify players' understanding that they have no reason to consider unconventional alternatives as long as others are unlikely to do so.

To formalize these ideas, we have embedded the game in a larger game of incomplete information, in which players may randomly and independently consider any nonempty subset of their actual strategy set in the game, and where a player's actual consideration set, that is the player's "type," is his or her private information. We defined a coarsely tenable strategy block as a convention such that there could never be any advantage for any player to consider any unconventional strategy in any population equilibrium in which the probability of other players being conventional, that 
is, considering precisely the given conventional block, is close to one. This seemed a good basic definition of "external stability" of a conventional block of strategies. We obtained "internal stability" by focusing on minimal coarsely tenable blocks, that is coarsely tenable blocks that do not contain other coarsely tenable blocks that could become conventions by themselves. However, defining coarsely settled equilibria as Nash equilibria with support in minimal coarsely tenable blocks, we noted that in some games this approach failed to exclude some Nash equilibria that seemed unreasonable to us, and so we developed a concept of fine tenability that would admit smaller tenable blocks.

Our concept of fine tenability was derived by restricting the robustness test to a smaller class of type distributions around any given block. More precisely, we focused on those type distributions in which any unconventional player would be much more likely to consider a larger than a smaller subset of the strategies that are available in his or her player role in the actual game (with "large" and "small" defined in terms of set inclusion). This restriction on the type distribution thus places more probability on "more rational" unconventional types than on "less rational" unconventional types. We accordingly defined a finely tenable strategy block as a convention such that there could never be any advantage for any player to consider any unconventional strategy in any population equilibrium in which the probability of other players being conventional is close to one, and where the type distribution is "rationality biased" in the said sense. This gives a slightly weaker definition of "external stability" of a conventional block of strategies. We obtained "internal stability" also for this version by focusing on minimal finely tenable blocks, and we defined finely settled equilibria as Nash equilibria with support in such blocks. While every coarsely tenable block is also finely tenable, a coarsely settled equilibrium need not be finely settled, and a finely settled equilibrium need not be coarsely settled. We call equilibria with both properties fully settled. Every finite game admits at least one fully settled equilibrium. Moreover, in generic normal-form games the coarsely tenable blocks coincide with the finely tenable blocks and thus also the three versions of settledness generically coincide.

By way of examples, we showed the nature and power of these solution concepts. In particular, the rejection of the mixed equilibrium in the coordination game in the introduction was shown to hold even when one or both of the zero-payoff outcomes was replaced by a zero-sum game, and also when the mixed Nash equilibrium strategy was added as a third pure strategy. In other examples we showed that minimal tenable blocks and settled equilibria make well-behaved and sharp predictions in some games where standard refinements usually fail. The difference was particularly stark in a signaling game and in a voting game. In the battle-of-the-sexes game with an outside-option, the forward-induction solution was seen to be the only fully settled equilibrium, while the "outside option" equilibrium component contained another 
finely (but not coarsely) settled equilibrium. Yet other examples showed that the present approach makes sharper predictions than curb and persistence in an open set of games. A final example demonstrated that there are games in which tenable blocks overlap.

We here analyzed general type distributions (for coarse tenability) and "rationality biased" type distributions (for fine tenability) around a conventional strategy block. However, also other restrictions on the type distribution might be worth studying. Any other restriction (within the general class admitted in our definition of coarse tenability) could yield another weak concept of tenability (compared with coarse tenability) which in turn could be used to define associated concepts of settled equilibria. For example, in games with very large strategy sets (such as chess) it is unrealistic to assume that a player is able to consider his or her full strategy set. In such games "rationality biased" type distributions may thus be empirically irrelevant, and it might be more relevant to instead consider "behavioral" type distributions that attach little probability to very large consideration sets. We would look forward to future research on alternative restrictions on type distributions around conventional blocks.

Our concepts of tenability also permit evolutionary interpretations. A (pure or mixed) strategy in a finite and symmetric two-player game is neutrally (or weakly evolutionarily) stable if no other strategy does better in the post-entry population mix that arises under uniform random matching in a large population playing the game, where almost everybody plays the original "incumbent" strategy and only a few individuals play the new "mutant" strategy. Any symmetric coarsely tenable singleton block has this robustness property. To see the connection between neutral stability and tenability more generally, consider any block $T$ in any finite game $G$. Suppose that the game is played recurrently in a large population, with individuals randomly and uniformly drawn from (infinitely) large player populations, one for each player role, just as in Nash's (1950) mass-action interpretation. Suppose that initially all individuals only use strategies in the block $T$ under consideration. For each player role $i$, let $\sigma_{i}$ be any "mutant" strategy that suddenly appears in a small population share $\varepsilon$ in player population $i$. The introduction of such mutant strategies could be interpreted as a game with random consideration sets where, in each population $i$, a $1-\varepsilon$ fraction are "normal" individuals, the "incumbents," who consider the set $T_{i}$ but, for each pure strategy $s_{i}$, an $\varepsilon \sigma_{i}\left(s_{i}\right)$ fraction are mutants who consider only $\left\{s_{i}\right\}$. If the block $T$ is coarsely tenable, no mutant will fare better than the normal players (in the same player population) when all normal players respond rationally within their consideration sets. It would be interesting to further explore connections between the present solution concepts and those in evolutionary game theory, for instance comparing fine tenability with the set-valued notions of robustness to equilibrium 
entrants in Swinkels $(1992 a, b) .{ }^{21}$

The present approach also suggests other avenues for further research, where the most evident appears to be to apply the present machinery to well-known (finite) games that represent important interactions in economics, political science and other social and behavior sciences. Do the solutions suggested here match up with what we know or believe about the likely outcomes in such interactions? For example, games with cheap talk, signalling or voting usually have plethora of Nash equilibria, many of which are arguably unreasonable as predictions of actual behavior, and yet standard refinements have little cutting power. We conjecture that the present machinery, by contrast, may have a lot of cutting power in such games. ${ }^{22} \mathrm{~A}$ second avenue would be to study the solutions' predictive power in controlled laboratory experiments. Will human subjects in the lab, under random rematching and with some opportunity for social learning, tend towards minimal tenable blocks and settled equilibria? A third avenue could be to explore connections between our solutions and explicit models of population dynamics. There is a handful such models in the economics literature. Some of these have been shown to converge to minimal curb sets, see Young (1993, 1998), Hurkens (1995) and Sanchirico (1996). In the same vein, Kets and Voorneveld (2008) establish convergence to minimal prep sets (see Remark 1) when individuals tend to favor recently used strategies - a form of habit formation. It is also known from the literature on dynamic learning and evolution in games (see e.g. Nachbar, 1990, and Weibull, 1995) that if such a process meets certain regularity conditions, and if it converges, then the limit point will necessarily be a Nash equilibrium. In such dynamic population models, will settled equilibria and minimal tenable blocks be good predictors?

\footnotetext{
${ }^{21}$ Loosely speaking, while Swinkels requires robustness against entrants who play post-entry optimal strategies when incumbents do not adjust their behavior, fine tenability requires robustness against rationality-biased mutant type distributions when incumbents optimally adjust their behavior within the conventional block.

${ }^{22}$ Laslier and Van den Straaten (2004) show that while perfection has virtually no cutting power in a class of voting games, "true perfecting" (see Kalai and Samet, 1984) effectively eliminates implausible equilibria. The present machinery may lead to similarly sharp conclusions, since tenability requires robustness to a wide range of type distributions much in the same way as true perfection requires robustness to a wide range of strategy perturbations.
} 


\section{REFERENCES}

[1] Balkenborg, D., J. Hofbauer, and C. Kuzmics (2013): "Refined best-response correspondence and dynamics", Theoretical Economics 8, 165-192.

[2] Balkenborg, D., J. Hofbauer, and C. Kuzmics (2014): "The refined best-response correspondence in normal form games", mimeo., Department of Economics, University of Exeter.

[3] Basu, K., and J. Weibull (1991): "Strategy subsets closed under rational behavior", Economics Letters 36, 141-146.

[4] Battigalli, P. and M. Siniscalchi (2002): "Strong belief and forward induction reasoning", Journal of Economic Theory 106, 356-391.

[5] Battigalli, P. and A. Friedenberg (2012): "Forward induction revisited", Theoretical Economics 7, 57-98.

[6] Bernheim, D. (1984): "Rationalizable strategic behavior", Econometrica 52, 1007-1028.

[7] Brandenburger, A. and E. Dekel (1987): "Rationalizability and correlated equilibria", Econometrica 55, 1391-1402.

[8] Brandenburger, A., A. Friedenberg and H.J. Keisler (2008): "Admissibility in games", Econometrica 76, 307-352.

[9] Brandenburger, A. and A. Friedenberg (2010): "Self-admissible sets", Journal of Economic Theory 145, 785-811.

[10] Eliaz, K., and R. Spiegler (2011): "Consideration sets and competitive marketing", Review of Economic Studies 78, 235-262.

[11] Gordon, S. (2011): "Iteratively stable cheap talk, Department of Economics", Ecole des Sciences Politiques, Paris.

[12] Halpern, J., and R. Pass (2009): "Algorithmic rationality: game theory with costly computation", Working Paper, Department of Computer Science, Cornell University.

[13] Harsanyi, J. (1973): "Oddness of the number of equilibrium points: a new proof", International Journal of Game Theory 2, 235-250.

[14] Hurkens, S. (1995): "Learning by forgetful players", Games and Economic Behavior 11, 304-329. 
[15] Hurwicz, L. (2008): "But who will guard the guardians?", American Economic Review 98, 577-585.

[16] Jansen, M. (1981): "Regularity and stability of equilibrium points of bimatrix games", Mathematics of Operations Research 6, 530-550.

[17] Kalai, E. and D. Samet (1984): "Persistent equilibria in strategic games", International Journal of Game Theory 13, 129-144.

[18] Kets, W. and M. Voorneveld (2008): "Learning to be prepared", International Journal of Game Theory 37, 333-352.

[19] Kohlberg, E. and J.-F. Mertens (1986): "On the strategic stability of equilibria", Econometrica 54, 1003-1037.

[20] Laslier, J.-F. and K. Van den Straaten (2004): "Electoral competition under imperfect information", Economic Theory 24, 419-446.

[21] Lewis, D. (1969): Convention: A Philosophical Study. Harvard University Press, Cambridge MA, USA.

[22] Mailath, G., L. Samuelson and J. Swinkels (1993): "Extensive form reasoning in normal form games", Econometrica 61, 273-302.

[23] Manzini, P. and M. Mariotti (2007): "Sequentially rationalizable choice", American Economic Review 97, 1824-1839.

[24] Manzini, P. and M. Mariotti (2014): "Stochastic choice and consideration sets", Econometrica 82, 1153-1176.

[25] Myerson, R. (1978): "Refinements of the Nash equilibrium concept", International Journal of Game Theory 7, 73-80.

[26] Myerson, R. (1996): "Sustainable equilibria in culturally familiar games", in W. Albers et al. (eds.), Understanding Strategic Interaction: Essays in Honor of Reinhard Selten. Springer Verlag, Berlin.

[27] Nachbar, J. (1990): "Evolutionary selection dynamics in games: convergence and limit properties", International Journal of Game Theory 19, 59-89.

[28] Nash, J.F. (1950): Non-Cooperative Games, Ph.D. thesis, Princeton University. Reprinted in H. Kuhn and S. Nasar (2002), The Essential John Nash, Princeton University Press, 53-84. 
[29] Pearce, D. (1984): "Rationalizable strategic behavior and the problem of perfection", Econometrica 52, 1029-1050.

[30] Riker, W. (1982): "The two-party system and Duverger's law: an essay on the history of political science", American Political Science Review 76, 753-766.

[31] Ritzberger, K. and J. Weibull (1995): "Evolutionary selection in normal-form games", Econometrica 63, 1371-1399.

[32] Salant, Y. and A. Rubinstein (2008): "Choice with frames", Review of Economic Studies 75, 1287-1296.

[33] Sanchirico, C. (1996): "A probabilistic model of learning in games", Econometrica $64,1375-1394$.

[34] Schelling, T. (1960): The Strategy of Conflict. Harvard University Press, Cambridge MA, USA.

[35] Swinkels, J. (1992a): "Evolutionary stability with equilibrium entrants", Journal of Economic Theory 57, 306-332.

[36] Swinkels, J. (1992b): "Evolution and strategic stability: from Maynard Smith to Kohlberg and Mertens", Journal of Economic Theory 57, 333-342.

[37] Tan, T. and S. Werlang (1988): "The Bayesian foundations of solution concepts of games", Journal of Economic Theory 45, 370-395.

[38] Tercieux, O. (2006a): "p-Best response set”, Journal of Economic Theory 131, $45-70$.

[39] Tercieux, O. (2006b): "p-Best response set and the robustness of equilibria to incomplete information", Games and Economic Behavior 56, 371-384.

[40] van Damme, E. (1984): "A relation between perfect equilibria in extensive-form games and proper equilibria in normal-form games", International Journal of Game Theory 13, 1-13.

[41] van Damme, E. (1989): "Stable equilibria and forward induction", Journal of Economic Theory 48, 476-496.

[42] van Damme, E. (1991): Stability and Perfection of Nash Equilibrium (2nd ed.), Springer Verlag, Berlin.

[43] von Neumann J. and O. Morgenstern (1944): Theory of Games and Economic Behavior. Princeton University Press, Princeton, NJ. 
[44] Voorneveld, M. (2004): "Preparation", Games and Economic Behavior 48, 403414.

[45] Voorneveld, M. (2005): "Persistent retracts and preparation", Games and Economic Behavior 51, 228-232.

[46] Weibull, J. (1995): Evolutionary Game Theory, MIT Press, Cambridge, MA.

[47] Wright, C. and F. Barbour (1977): "Phased decision strategies: sequels to an initial screening". In M. Starr and M. Zeleny (eds.), Studies in Management Sciences, Multiple Criteria for Decision Making. North-Holland, Amsterdam.

[48] Wu, W. and J. Jiang (1962): "Essential equilibrium points of n-person noncooperative games", Scientia Sinica 11, 1307-1322.

[49] Young, P. (1993): "The evolution of conventions", Econometrica 61, 57-84.

[50] Young, P. (1998): Individual Strategy and Social Structure. Princeton University Press, Princeton, NJ. 